\title{
NONLINEAR ELLIPTIC BOUNDARY VALUE PROBLEMS FOR EQUATIONS WITH RAPIDLY (OR SLOWLY) INCREASING COEFFICIENTS
}

\author{
BY \\ JEAN-PIERRE GOSSEZ
}

\begin{abstract}
Variational boundary value problems for quasilinear elliptic systems in divergence form are studied in the case where the nonlinearities are nonpolynomial. Monotonicity methods are used to derive several existence theorems which generalize the basic results of Browder and Leray-Lions. Some features of the mappings of monotone type which arise here are that they act in nonreflexive Banach spaces, that they are unbounded and not every where defined, and that their inverse is also unbounded and not every where defined.
\end{abstract}

Introduction. This paper is concerned with the existence of solutions for variational boundary value problems for quasilinear elliptic systems in divergence form

$$
A(u) \equiv \sum_{|\alpha| \leq m}(-1)^{|\alpha|} D^{\alpha} A_{\alpha}\left(x, u, \cdots, \nabla^{m} u\right)
$$

on open subsets $\Omega$ of $\mathbf{R}^{n}$. Existence theorems for problems of this type were first obtained by Višik [34], [35] using compactness arguments and a priori estimates on $(m+1)$ st derivatives. Since 1963, these problems have been extensively studied by Browder and others in the context of the theory of mappings of monotone type from a reflexive Banach space to its dual and in the case where the coefficients $A_{a}$ have polynomial growth in $u$ and its derivatives. Basic improvements of Browder's original results [3] were given by Leray-Lions [25] where the monotonicity conditions imposed on $A$ involve only the variation of $A_{a},|a|=m$, with respect to the top order derivatives $\nabla^{m} u$, and by Browder [7], [8] where the usual coercivity assumption is replaced either by a local a priori bound and a stronger monotonicity condition or by a global a priori bound and some oddness condition.

It is our purpose here tq extend the existence theorems of [3], [25], [7], [8]

Presented to the Society, April 1, 1972 under the title Quasilinear elliptic equations with rapidly increasing coefficients; received by the editors December 2, 1972.

AMS (MOS) subject classifications (1970). Primary 47H05, 35K65; Secondary $46 \mathrm{~B} 10$.

Key words and phrases. Quasilinear elliptic systems, nonpolynomial nonlinearities, existence theorems, nonlinear monotone mappings, nonlinear pseudomonotone mappings, Orlicz-Sobolev spaces, nonreflexive Banach spaces, complementary systems, noncoercive problems, local a priori bound. 
to the case where the coefficients $A_{a}$ do not necessarily have polynomial growth in $u$ and its derivatives. The crucial points in the treatment of "rapidly (or slowly) increasing" $A_{a}$ 's are that the Banach spaces in which the problems seem to be appropriately formulated-the Orlicz-Sobolev spaces-are not reflexive and that the corresponding mappings of monotone type are not bounded nor everywhere defined and do not generally satisfy a global a priori bound (and consequently are not generally coercive). In this respect, the examination of the trivial situation where $m=0$ is already quite revealing (see Examples 2.3 and 3.13). Our study is based upon an extension of the theory of non everywhere defined unbounded pseudomonotone mappings in reflexive Banach spaces (Browder [9], [10], Browder-Hess [11]) to the context of complementary systems. These are quadruples of (generally nonreflexive nonseparable) normed spaces related to each other in roughly the same way as conjugate Orlicz spaces.

A simple example to which our results can be applied is the Dirichlet problem for the operator

$$
\sum_{|a|=m}(-1)^{|a|} D^{\alpha}\left(p\left(D^{a} u\right)\right)+\text { lower order terms, }
$$

where $p: \mathbf{R} \rightarrow \mathbf{R}$ is any strictly increasing odd continuous function going to $+\infty$ at $+\infty$, with no restrictions on its growth, and where the lower order terms satisfy a growth condition involving $p$ and a sign condition (see Example 5.6).

Monotonicity methods have previously been used to study systems of the form (*) with rapidly increasing coefficients by Donaldson [12] (cf. also Gossez [17]) who treated the case where the problem is coercive, the $A_{a}$ 's satisfy a monotonicity condition with respect to all the derivatives of $u$ and some restriction is imposed on the nature of the growth of the coefficients (the conjugate $N$-functions are required to have the $\Delta_{2}$ property). Each of these three limitations is removed or weakened in the present paper. Our results also include and sharpen the existence theorem announced recently by Fougères [15] where the second limitation above is weakened. Last year Browder [0] (cf. also Hess [22], [23]) considered șystems of the form (*) with top order terms of polynomial growth but "strongly nonlinear" lower order terms. Our results will be generalized elsewhere so as to include this situation (see Example 4.12).

In $\$ 1$ we define the notion of complementary system and give some important examples. Pseudomonotone mappings in complementary systems are considered in $\$ 2$ and several of their properties are investigated. In $\$ 3$ we prove our main existence theorems for functional equations involving pseudomonotone mappings in complementary systems. They are applied to systems of the form $(*)$ with rapidly (or slowly) increasing coefficients in $\$ \$ 4$ and 5 .

Some of the results presented here were announced in [19]. The author would 
like to thank Professor F. E. Browder for several stimulating conversations, Professor M. Jodeit for his help in the proof of Lemma 4.14, and the referee for suggesting a simple proof of Lemmas 1.5, 1.6 and 1.7.

1. Complementary systems. The usual functional setting $\left(X, X^{*}\right)$ consisting of a reflexive Banach space $X$ and its dual $X^{*}$ does not seem suitable for the study of systems of the form (*) with rapidly (or slowly) increasing coefficients. In this section we consider a more general setting, that of complementary systems, and prove that the Orlicz-Sobolev spaces generate complementary systems.

We begin by listing briefly some definitions and well-known facts from Orlicz space theory (cf. [24], [28]). Let $\Omega$ be an open subset of $R^{n}$, with Lebesgue measure $d x$, and let $M$ be a $N$-function (i.e. a real-valued continuous, convex, even function of $t \in \mathbf{R}$ satisfying $M(t)>0$ for $t>0, M(t) / t \rightarrow 0$ as $t \rightarrow 0$ and $M(t) / t \rightarrow+\infty$ as $t \rightarrow+\infty)$. The Orlicz class $\mathscr{L}_{M}(\Omega)$ is defined as the set of (equivalence classes of) real-valued measurable functions $u$ on $\Omega$ such that $\int_{\Omega} M(u(x)) d x<+\infty$, and the Orlicz space $L_{M}(\Omega)$ as the linear hull of $\mathscr{L}_{M}(\Omega)$. $L_{M}(\Omega)$ is a Banach space with respect to the Luxemburg norm

$$
\|u\|_{(M)}=\inf \left\{k>0 ; \int_{Q} M(u / k) d x \leq 1\right\} \text {. }
$$

The closure in $L_{M}(\Omega)$ of the bounded measurable functions with compact support in $\bar{\Omega}$ is denoted by $E_{M}(\Omega)$. The inclusions $E_{M}(\Omega) \subset \mathscr{Q}_{M}(\Omega) \subset L_{M}(\Omega)$ hold. Moreover, $E_{M}(\Omega)=\mathscr{L}_{M}(\Omega)$ if and only if $\mathscr{L}_{M}(\Omega)=L_{M}(\Omega)$ if and only if $M$ has the $\Delta_{2}$ property for large values of $t$, or for all values of $t$, according to whether $\Omega$ has finite measure or not, i.e., there exists $k>0$ such that $M(2 t) \leq k M(t)$ for large values of $t$, or for all values of $t . E_{M}(\Omega)$ is separable, but $L_{M}(\Omega)$ is separable if and only if $L_{M}(\Omega)=E_{M}(\Omega)$. The dual of $E_{M}(\Omega)$ can be identified by means of the scalar product $\int_{\Omega} u v d x$ to $L_{M}(\Omega)$, where $\bar{M}$ is the $N$-function conjugate to $M$ :

$$
\bar{M}(t)=\sup \{t s-M(s) ; s \in \mathbf{R}\} .
$$

Note that $\overline{\bar{M}}=M$ and that Young's inequality holds: $t s \leq M(t)+\bar{M}(s)$ for all $t, s$ $\epsilon$ R. The norm on $L_{M}(\Omega)$ dual to \|\|$_{(M)}$ on $E_{M}(\Omega)$ is called the Orlicz norm and denoted by \|\|$_{M^{*}}$ It is equivalent to \|\|$_{(M)}$ : \|\|$_{(M)} \leq\|\|_{M} \leq 2\|\|_{(M)}$. The norm on $L_{M}(\Omega)$ dual to \|\|$_{M}$ on $E_{M}(\Omega)$ turns out to be \|\|$_{(M)}$. Hölder's inequality holds:

$$
\int_{\mathbf{Q}} u v d x \leq\|u\|_{M}\|v\|_{(\bar{M})}
$$

for all $u \in L_{M}(\Omega)$ and $v \in L_{M}(\Omega)$.

Definition 1.1. Let $Y$ and $Z$ be real Banach spaces in duality with respect 
to a continuous pairing $\langle$,$\rangle and let Y_{0}$ and $Z_{0}$ be subspaces of $Y$ and $Z$ respectively. Then $\left(Y, Y_{0} ; Z, Z_{0}\right)$ is called a complementary system if, by means of $\langle\rangle,, Y_{0}^{*}$ can be identified (i.e., is linearly homeomorphic) to $Z$ and $Z_{0}^{*}$ to $Y$.

For instance $\left(L_{M}(\Omega), E_{M}(\Omega) ; L_{\bar{M}}(\Omega), E_{\bar{M}}(\Omega)\right)$ is a complementary system. More general examples arise in the theory of Banach function spaces [28]. Other examples are $\left(X^{* *}, X ; X^{*}, X^{*}\right)$ and $\left(X^{*}, X^{*} ; X^{* *}, X\right)$ where $X$ is a Banach space. Note that in a complementary system, $Y_{0}$ is $\sigma(Y, Z)$ dense in $Y$. Note also that if $\mathrm{cl} Y_{0}\left[\mathrm{cl} Z_{0}\right]$ denotes the (norm) closure of $Y_{0}\left[Z_{0}\right]$ in $Y[Z]$, then $\left(Y, \mathrm{cl} Y_{0} ; Z, \mathrm{cl} Z_{0}\right)$ is a complementary system.

It will be important in the applications to know a method by which given a complementary system $\left(Y, Y_{0} ; Z, Z_{0}\right)$ and a closed subspace $E$ of $Y$, one can construct a new complementary system $\left(E, E_{0} ; F, F_{0}\right)$. Some restriction must be imposed on $E$. Define $E_{0}=E \cap Y_{0}, F=Z / E_{0}^{\perp}$ and $F_{0}=\left\{z+E_{0}^{\perp} ; z \in Z_{0}\right\} \subset F$, where 1 denotes the orthogonal in the duality $(Y, Z)$, i.e. $E_{0}^{\perp}=\{z \in Z ;\langle y, z\rangle=0$ for all $y \in E_{0}$ \}.

Lemma 1.2. The pairing $\langle$,$\rangle between Y$ and $Z$ induces a pairing between $E$ and $F$ if and only if $E_{0}$ is $\sigma(Y, Z)$ dense in $E$. In this case, $\left(E, E_{0} ; F, F_{0}\right)$ is a complementary system if $E$ is $\sigma\left(Y, Z_{0}\right)$ closed, and conversely, when $Z_{0}$ is complete, $E$ is $\sigma\left(Y, Z_{0}\right)$ closed if $\left(E, E_{0} ; F, F_{0}\right)$ is a complementary system.

Proof. The pairing between $Y$ and $Z$ induces a pairing between $E$ and $F$ if and only if $E \subset E_{0}^{\perp \perp}$, so that the first part of the lemma follows from the bipolar theorem. The pairing between $E$ and $F$ obtained in this way is continuous and $E_{0}^{*}$ can be identified to $F$. To prove that $F_{0}^{*}$ can be identified to $E$ when $E$ is $\sigma\left(Y, Z_{0}\right)$ closed, define a mapping $A$ from $E$ into $F_{0}^{*}$ by

$$
(A y)\left(z+E_{0}^{\perp}\right)=\left\langle y, z+E_{0}^{\perp}\right\rangle_{E, F}=\langle y, z\rangle_{Y, Z}
$$

for $Y \in E$ and $z \in Z_{0} . A$ is linear, continuous and one-to-one. Let $L \in F_{0}^{*}$ and consider the continuous linear form on $Z_{0} /\left(E_{0}^{\perp} \cap Z_{0}\right)$ :

$$
z+\left(E_{0}^{\perp} \cap Z_{0}\right) \rightarrow L\left(z+E_{0}^{\perp}\right)
$$

for $z \in Z_{0}$. Since the dual of $Z_{0} /\left(E_{0}^{\perp} \cap Z_{0}\right)$ can be identified to $\left(E_{0}^{\perp} \cap Z_{0}\right)^{\perp}=$ $\sigma\left(Y, Z_{0}\right) c l E_{0}=E$, we conclude that there exists $y \in E$ such that $A y=L$. Consequently $A$ is onto, and by the closed graph theorem, $A$ is a linear homeomorphism between $E$ and $F_{0}^{*}$. Conversely, suppose now that $A$ is onto and that $Z_{0}$ is complete. To show that $E$ is $\sigma\left(Y, Z_{0}\right)$ closed, it suffices, by the KreinŚmulian theorem [14, p. 429], to prove that the limit $y \in Y$ of a bounded $\sigma\left(Y, Z_{0}\right)$ convergent net $y_{i} \in E$ lies in $E$. But the bounded sets of $E$ are relatively compact for $\sigma\left(E, Z_{0}\right)$ because $A$ transforms a bounded set of $E$ into a bounded set 
of $F_{0}^{*}$ and $A^{-1}$ is continuous from the weak* topology of $F_{0}^{*}$ to $\sigma\left(E, Z_{0}\right)$. Consequently $y \in E$. Q.E.D.

We will refer to the complementary system $\left(E, E_{0} ; F, F_{0}\right)$ constructed above as the complementary system generated by $E$ in $\left(Y, Y_{0} ; Z, Z_{0}\right)$. Note that $\sigma(E, F)$ and $\sigma\left(E, F_{0}\right)$ are the topologies induced on $E$ by $\sigma(Y, Z)$ and $\sigma\left(Y, Z_{0}\right)$ respectively, so that $F_{0}$ is precisely the subspace of $E_{0}^{*}$ consisting of those linear forms on $E_{0}$ which are $\sigma\left(Y_{0}, Z_{0}\right)$ continuous. The situation is much simpler when $Z=Z_{0}$ since then Lemma 1.2 can be applied to the $\sigma\left(Y, Z_{0}\right)$ closure of any subspace of $Y_{0}$.

The definition of a complementary system was first given by Donaldson [12], [13], but his analogue of Lemma 1.2 appears incorrect. Variants were also considered in a reflexive setting by Hess [22].

Let $W^{m} L_{M}(\Omega)$ be the Orlicz-Sobolev space of functions $u$ such that $u$ and its distribution derivatives up to order $m$ lie in $L_{M}(\Omega)$. $W^{m} L_{M}(\Omega)$ is a Banach space with respect to the norm

$$
\|u\|_{m, M}=\left(\sum_{|a| \leq m}\left\|D^{\alpha} u\right\|_{(M)}^{2}\right)^{1 / 2} .
$$

$W^{m} L_{M}(\Omega)$ will always be identified to a subspace of the product $\Pi_{|a| s m} L_{M}(\Omega) \equiv$ $\Pi L_{M}$; this subspace is $\sigma\left(\Pi L_{M}, \Pi E_{\bar{M}}\right)$ closed. Let $W_{0}^{m} L_{M}(\Omega)$ be the $\sigma\left(\Pi L_{M}, \Pi E_{\bar{M}}\right)$ closure of $\mathscr{D}(\Omega)$ in $W^{m} L_{M}(\Omega)$. We wish to apply Lemma 1.2 to $W^{m} L_{M}(\Omega)$ and $W_{0}^{m} L_{M}(\Omega)$, starting with the complementary system (I $L_{M}, \Pi E_{M}$; II $L_{\bar{M}}$, $\Pi E_{\bar{M}}$ ). This is possible under the mild assumption that $\Omega$ has the segment property (i.e. there exist a locally finite open covering $\left\{O_{i}\right\}$ of $\partial \Omega$ and corresponding vectors $\left\{y_{i}\right\}$ such that for $x \in \bar{\Omega} \cap O_{i}$ and $0<t<1, x+t y_{i} \in \Omega$ ):

Theorem 1.3. Suppose that $\Omega$ bas the segment property. Then (a) $\mathcal{T}(\bar{\Omega})$ is $\sigma\left(\Pi L_{M}, \Pi L_{\bar{M}}\right)$ dense in $W^{m} L_{M}(\Omega),(\mathrm{b}) \mathfrak{D}(\Omega)$ is $\sigma\left(\Pi L_{M}, \Pi L_{\bar{M}}\right)$ dense in $W_{0}^{m} L_{M}(\Omega)$.

$\mathscr{D}(\bar{\Omega})$ denotes the restrictions to $\Omega$ of the functions in $\mathscr{D}\left(\mathbf{R}^{n}\right)$. Thus, when $\Omega$ has the segment property, $W^{m} L_{M}(\Omega)$ and $W_{0}^{m} L_{M}(\Omega)$ generate complementary systems in ( $L_{M}, \Pi E_{M} ; \Pi L_{\bar{M}}, \Pi E_{\bar{M}}$ ). The proof of Theorem 1.3 is based on the following lemmas.

Lemma 1.4. Let $u_{b} \in \mathscr{L}_{M}\left(\mathbf{R}^{n}\right)$ satisfy $u_{b} \rightarrow u$ a.e. in $\mathbf{R}^{n}$ and $M\left(u_{b}\right) \leq w_{b}$ a.e. in $\mathbf{R}^{n}$, where $w_{b} \rightarrow w$ in $L^{1}\left(\mathbf{R}^{n}\right)$. Then $u \in \mathcal{L}_{M}\left(\mathbf{R}^{n}\right)$ and $u_{b} \rightarrow u$ for $\sigma\left(L_{M}\left(\mathbf{R}^{n}\right), L_{\bar{M}}\left(\mathbf{R}^{n}\right)\right)$.

Proof. By Fatou's lemma, $u \in \mathfrak{L}_{M}\left(\mathbf{R}^{n}\right)$. It is sufficient to show that, for all $v \in \mathscr{Q}_{\bar{M}}\left(\mathbf{R}^{n}\right), u_{b} v \rightarrow u v$ in $L^{1}\left(\mathbf{R}^{n}\right)$. By contradiction, assume that for some $v \epsilon$ $\mathcal{L}_{\bar{M}}\left(\mathbf{R}^{n}\right), \delta>0$ and subsequence $b_{k}$, . 


$$
\int_{R^{n}}\left|u_{b_{k}} v-u v\right| d x \geq \delta
$$

Since $w_{b_{k}} \rightarrow w$ in $L^{1}\left(R^{n}\right)$, there is a subsequence, again denoted by $b_{k}$, and $g \in L^{1}\left(\mathbf{R}^{n}\right)$ such that $w_{b_{k}} \leq g$ a.e. in $\mathbf{R}^{n}$ (see e.g. N. Bourbaki, Intégration, Chapitre IV, $\$ 3$, Théorème 3). Therefore $\left|u_{b_{k}} v\right| \leq g+\bar{M}(v)$ by Young's inequality, and by Lebesgue's theorem, $u_{b_{k}} v \rightarrow u v$ in $L^{1}\left(R^{n}\right)$, a contradiction. Q.E.D.

Lemma 1.5. Let $u \in L_{M}\left(\mathbf{R}^{n}\right)$ and denote by $u_{y}$ the translated function: $u_{y}(x)=u(x-y)$. Then $u_{y} \rightarrow u$ for $\sigma\left(L_{M}\left(\mathbf{R}^{n}\right), L_{\bar{M}}\left(\mathbf{R}^{n}\right)\right)$ as $|y| \rightarrow 0$.

Proof. We can assume without loss of generality that $u \in \mathscr{L}_{M}\left(\mathbf{R}^{n}\right)$. Since $M\left(u_{y}\right)=M(u)_{y}$ converges to $M(u)$ in $L^{1}\left(\mathbf{R}^{n}\right)$, Lemma 1.5 follows from Lemma 1.4 . Q.E.D.

Lemma 1.6. Let $u \in L_{M}\left(\mathbf{R}^{n}\right)$ and denote by $u_{\epsilon}$ the regularized function: $u_{\epsilon}=$ $u * \phi_{\epsilon}$, where $\phi_{\epsilon} \in \mathcal{D}\left(\mathbf{R}^{n}\right)$, bas support in $B_{\epsilon}\left(0, R^{n}\right)$ and satisfies $\phi_{\epsilon} \geq 0$ and $\int_{\mathbf{R}^{n}} \phi_{\epsilon}(x) d x=1$. Then $u_{\epsilon} \rightarrow u$ for $\sigma\left(L_{M}\left(\mathbf{R}^{n}\right), L_{\bar{M}}\left(\mathbf{R}^{n}\right)\right)$ as $\epsilon \rightarrow 0$.

Proof. We can assume without loss of generality that $u \in \mathcal{L}_{M}\left(\mathbf{R}^{n}\right)$. By Jensen's inequality, $M\left(u_{\epsilon}\right) \leq M(u) * \phi_{\epsilon}$. Since $M(u) * \phi_{\epsilon} \rightarrow M(u)$ in $L^{1}\left(\mathbf{R}^{n}\right)$, Lemma 1.6 follows from Lemma 1.4. Q.E.D.

Lemma 1.7. Let $u \in L_{M}(\Omega)$ and denote by $u_{r}$ the function $u_{r}=u \psi_{r}$, where $\psi_{r}(x)=\psi(x / r)$ and $\psi \in \mathscr{D}\left(\mathbf{R}^{n}\right)$ satisfies $0 \leq \psi \leq 1, \psi(x)=1$ for $|x| \leq 1$ and $\psi(x)=0$ for $|x| \geq 2$. Then $u_{r} \rightarrow u$ for $\sigma\left(L_{M}(\Omega), L_{\bar{M}}(\Omega)\right)$ as $r \rightarrow \infty$

Proof. We can assume without loss of generality that $u \in \mathscr{Q}_{M}(\Omega)$. Since $M\left(u_{r}\right) \leq \psi_{r} M(u)$ and $\psi_{r} M(u) \rightarrow M(u)$ in $L^{1}(\Omega)$, Lemma 1.7 follows from Lemma 1.4. Q.E.D.

Remark 1.8. The weaker versions of the last three lemmas where $\sigma\left(L_{M}, L_{\bar{M}}\right)$ is replaced by $\sigma\left(L_{M}, E_{\bar{M}}\right)$ follow easily by transposition from the fact (cf. e.g. [13]) that if $u \in E_{M}\left(\mathbf{R}^{n}\right)$, then $u_{y^{\prime}} u_{\epsilon}$ and $u_{r}$ lie in $E_{M}\left(\mathbf{R}^{n}\right)$ and converge in norm to $u$ as $|y|, \epsilon$ and $1 / r \rightarrow 0$.

Proof of Theorem 1.3. The proof uses arguments which are standard in Sobolev space theory (cf. e.g. [1, pp. 11-14]) and we only sketch it.

Let $u \in W^{m} L_{M}(\Omega)$. Using Lemma 1.7, we can assume that $u$ has compact support $K \subset \bar{\Omega}$. If $K \subset \Omega$, then the conclusion can be derived from Lemma 1.6. If $K$ meets $\partial \Omega$, then, using the covering $\left\{O_{i}\right\}$ of $\partial \Omega$ and a partition of unity, we are reduced to the case $K \subset O_{i}$ for some $i$. Clearly, $K \subset O_{i}^{\prime}$ for some open set $O_{i}^{\prime}$ with compact closure $\bar{O}_{i}^{\prime}$ in $O_{i^{\bullet}}$ Write $\Gamma=\bar{O}_{i}^{\prime} \cap 2 \Omega, \Gamma_{t}=\Gamma-t y_{i}$ where $y_{i}$ is 
the vector associated with $O_{i}$ in the segment propertv and $0<t<$ $\min \left\{1,\left|y_{i}\right|^{-1} \operatorname{dist}\left(O_{i}^{\prime}, \partial O_{i}\right)\right\}$. Extend $u$ outside $K$ by zero and define $u_{t}(x)=$ $u\left(x+t y_{i}\right)$. Then $u_{t} \in W^{m} L_{M}\left(\mathbf{R}^{n} \backslash \Gamma_{t}\right)$, and by the segment property, dist $\left(\Gamma_{t}, \Omega\right)>0$. Using Lemma 1.5, we see that $u_{t} \rightarrow u$ in $W^{m} L_{M}(\Omega)$ for $\sigma\left(\Pi L_{M}, \Pi L_{\bar{M}}\right)$ as $t \rightarrow 0$, so that it suffices to approximate each $u_{t}$ by functions in $\mathscr{D}(\bar{\Omega})$. But this can be done by means of Lemma 1.6 because dist $\left(\Gamma_{t}, \Omega\right)>0$.

To prove part (b), first note that if $u \in W_{0}^{m} L_{M}(\Omega)$, then the function $\tilde{u}$ obtained by extending $u$ outside $\Omega$ by zero belongs to $W^{m} L_{M}\left(\mathbf{R}^{n}\right)$. Now let $u \epsilon$ $W_{0}^{m} L_{M}(\Omega)$. As above, we are reduced to the case $K \subset O_{i}$ for some $i$. Define $u_{t}(x)=\tilde{u}\left(x-t y_{i}\right)$. Then $u_{t} \in W^{m} L_{M}\left(\mathbf{R}^{n}\right)$ and supp $u_{t} \subset \Omega$ by the segment property. Moreover, using Lemma 1.5, we see that $u_{t} \rightarrow u$ in $W^{m} L_{M}(\Omega)$ for $\sigma\left(\Pi L_{M}, \Pi L_{\bar{M}}\right)$, so that it suffices to approximate each $u_{t}$ by functions in $\mathscr{T}(\Omega)$. But this can be done by means of Lemma 1.6 because supp $u_{t}$ is compact in $\Omega$. Q.E.D.

Remark 1.9. The above proof shows that the densities in Theorem 1.3 are sequential.

Let $W^{m} E_{M}(\Omega)$ be the space of functions $u$ such that $u$ and its distribution derivatives up to order $m$ lie in $E_{M}(\Omega)$, and $W_{0}^{m} E_{M}(\Omega)$ the (norm) closure of $D(\Omega)$ in $W^{m} L_{M}(\Omega)$. Clearly, $W^{m} E_{M}(\Omega)$ is the intersection of $W^{m} L_{M}(\Omega)$ with $\Pi E_{M}$ and $W_{0}^{m} E_{M}(\Omega) \subset W^{m} E_{M}(\Omega)$.

Corollary 1.10. If $\Omega$ bas the segment property, then (a) $\mathscr{D}(\bar{\Omega})$ is (norm) dense in $W^{m} E_{M}(\Omega)$, (b) $W_{0}^{m} E_{M}(\Omega)$ is the intersection of $W_{0}^{m} L_{M}(\Omega)$ with $\Pi E_{M}$.

Proof. By Theorem 1.3, $\mathscr{D}(\bar{\Omega})$ is dense in $W^{m} E_{M}(\Omega)$ for $\sigma\left(\Pi E_{M}, \Pi L_{\bar{M}}\right)$. Since $\Pi L_{\bar{M}}$ is the dual of $\Pi E_{M}$ and $\mathscr{D}(\bar{\Omega})$ is convex, $\mathscr{D}(\bar{\Omega})$ is norm dense in $W^{m} E_{M}(\Omega)$. The proof that $\mathscr{D}(\Omega)$ is norm dense in the intersection of $W_{0}^{m} L_{M}(\Omega)$ with $\Pi E_{M}$ is similar. Q.E.D.

Part (a) of Theorem 1.3 sharpens Theorem 4.1 of Donaldson-Trudinger [13] where $\sigma\left(\Pi L_{M}, \Pi L_{\bar{M}}\right)$ is replaced by $\sigma\left(\Pi L_{M}, \Pi E_{\bar{M}}\right)$. Part (a) of Corollary 1.10 was obtained in [13, Theorems 2.1 and 2.3]. When $\partial \Omega$ is sufficiently good, one can define a trace function from $W^{m} L_{M}(\Omega)$ into $W^{m-1} L_{M}(\partial \Omega)$ whose kernel in $W^{m} L_{M}(\Omega)\left[W^{m} E_{M}(\Omega)\right]$ is precisely $W_{0}^{m} L_{M}(\Omega)\left[W_{0}^{m} E_{M}(\Omega)\right]$ (see A. Fougères, C. R. Acad. Sci. Paris, January 1972, in the particular case where $\bar{M}$ has the $\Delta_{2}$ property).

We now investigate a property of the norm which will be useful later when dealing with the duality mapping. Let $\left(Y, Y_{0} ; Z, Z_{0}\right)$ be a complementary system and let \|\|$_{Y}$ be a (equivalent) norm on $Y$. Denote by \|\|$_{Y_{0}}$ the restriction of \|\|$_{Y}$ to $Y_{0}$, by \|\|$_{Z}$ the norm on $Z$ dual to \|\|$_{Y_{0}}$ and by \|\|$_{Z_{0}}$ the restriction of \|\|$_{Z}$ to $Z_{0}$. 
Lemma 1.11. The norm \|\|$_{Y}$ is dual to \|\|$_{Z_{0}}$ if and only if \|\|$_{Y}$ is $\sigma\left(Y, Z_{0}\right)$ lower semicontinuous and the ball $B_{1}\left(0, Y_{0}\right)$ is $\sigma\left(Y, Z_{0}\right)$ dense in $B_{1}(0, Y)$. The inequality $\langle y, z\rangle \leq\|y\|_{Y}\|z\|_{Z}$ bolds for all $y \in Y$ and $z \in Z$ if and only if $B_{1}\left(0, Y_{0}\right)$ is $\sigma(Y, Z)$ dense in $B_{1}(0, Y)$.

Proof. Since $B_{1}\left(0, Z_{0}\right)$ is the polar in $Z_{0}$ of $B_{1}\left(0, Y_{0}\right)$, the first part of the lemma follows from the bipolar theorem. The second part is an easy consequence of the Hahn-Banach theorem. Q.E.D.

A (equivalent) norm \|\|$_{Y}$ on $Y$ satisfying all the conditions of Lemma 1.11 will be called admissible. For instance, in the complementary system $\left(L_{M}(\Omega)\right.$, $E_{M}(\Omega) ; L_{\bar{M}}(\Omega), E_{\bar{M}}(\Omega)$ ), both the Luxemburg and the Orlicz norms are admissible.

Lemma 1.12. Let $\left(Y, Y_{0} ; Z, Z_{0}\right)$ be a complementary system and let \|\|$_{Y}$ be an admissible norm on $Y$. Let $E$ be a closed subspace of $Y$ satisfying the conditions of Lemma 1.2. Then the restriction \|\|$_{E}$ of \|\|$_{Y}$ to $E$ is admissible in the complementary system $\left(E, E_{0} ; F, F_{0}\right)$ generated by $E$ in $\left(Y, Y_{0} ; Z, Z_{0}\right)$.

Proof. Since $\sigma\left(Y, Z_{0}\right)$ induces $\sigma\left(E, F_{0}\right)$ on $E,\|\|_{E}$ is $\sigma\left(E, F_{0}\right)$ lower semicontinuous. The inequality $\langle e, f\rangle_{E, F} \leq\|e\|_{E}\|f\|_{F}$ for $e \in E$ and $f \in F$ follows from the definition of the quotient norm \|\|$_{F^{*}}$ Q.E.D.

Thus, when $\Omega$ has the segment property, the formula (1.1) and its analogue where \|\|$_{(M)}$ is replaced by \|\|$_{M}$ define admissible norms in the complementary systems generated by $W^{m} L_{M}(\Omega)$ and $W_{0}^{m} L_{M}(\Omega)$ in ( $L_{M}$, $\Pi E_{M} ; \Pi L_{\bar{M}}$, $\Pi E_{\bar{M}}$ ).

2. Mappings of monotone type. In this section pseudomonotonicity and the type (M) property are introduced for non everywhere defined unbounded mappings in complementary systems. Conditions are given under which the sum of two pseudomonotone mappings (or homotopies) is pseudomonotone. The example of the duality mapping is considered.

Definition 2.1. Let $\left(Y, Y_{0} ; Z, Z_{0}\right)$ be a complementary system and let $V$ be a dense subspace of $Y_{0}$. A mapping $T$ from $Y$ into $2^{Z}$ is said to be of type (M) with respect to $V$ if (a) $T$ is finitely continuous from $V$ to the $\sigma(Z, V)$ topology of $Z$ (i.e. $T y$ is a nonempty $\sigma(Z, V)$ compact convex subset of $Z$ for each $y \in V$ and $T$ is upper semicontinuous from each finite-dimensional subset of $V$ to the $\sigma(Z, V)$ topology of $Z)$, (b) for any net $\left(y_{i}, z_{i}\right)$ such that $z_{i} \in T y_{i}, y_{i} \epsilon$ $V, y_{i}$ bounded, $y_{i} \rightarrow y \in Y$ for $\sigma\left(Y, Z_{0}\right), z_{i} \rightarrow z \in Z$ for $\sigma(Z, V)$ and $\lim \sup \left\langle y_{i}, z_{i}\right\rangle \leq\langle y, z\rangle$, it follows that $z \in T y$. A mapping $T$ from $Y$ into $2^{Z}$ is said to be pseudomonotone with respect to $V$ if $(\mathrm{a})$ and $(\mathrm{b})^{\prime}$ hold, where (b) ${ }^{\prime} \equiv$ (b) except that one also requires $\left\langle y_{i}, z_{i}\right\rangle \rightarrow\langle y, z\rangle$ in the conclusion. More generally, a one-parameter family of mappings $T_{t}$ from $Y$ into $2^{Z}, t \in[0,1]$, is 
said to be a pseudomonotone bomotopy with respect to $V$ if (i) $T$ is finitely continuous from $[0,1] \times V$ to the $\sigma(Z, V)$ topology of $Z$, (ii) for any net $\left(t_{i}, y_{i}, z_{i}\right)$ such that $z_{i} \in T_{t_{i}}\left(y_{i}\right), t_{i} \rightarrow t, y_{i} \in V, y_{i}$ bounded, $y_{i} \rightarrow y \in Y$ for $\sigma\left(Y, Z_{0}\right)$, $z_{i} \rightarrow z \in Z$ for $\sigma(Z, V)$ and $\lim \sup \left\langle y_{i}, z_{i}\right\rangle \leq\langle y, z\rangle$, it follows that $z \in T_{t}(y)$ and $\left\langle y_{i}, z_{i}\right\rangle \rightarrow\langle y, z\rangle$.

Similar definitions can be given for sequentially of type $(M)$ with respect to $V$, sequentially pseudomonotone with respect to $V$ or sequentially pseudomonotone bomotopy with respect to $V$, where one requires (b), (b)' or (ii) to hold only for ordinary sequences. Note that all those definitions are invariant by translating $T$ or $T_{t}$ by a fixed element of $V$ or by adding to $T$ or $T_{t}$ a fixed element of $Z_{0}$.

In $\$ \$ 4$ and 5 we will show that under suitable assumptions on the coefficients, systems of the form (*) with rapidly (or slowly) increasing coefficients give rise to mappings of the above type. Other examples, related to the theory of monotone mappings in nonreflexive Banach spaces, will be mentioned in $\$ 3$.

Pseudomonotonicity and the type $(M)$ property were first defined by Brézis [2]. The extension of Brézis' original results to non everywhere defined unbounded mappings in reflexive Banach spaces was carried out by Browder [9], [10] and Browder-Hess [11]. The concept of pseudomonotone homotopy is due to Browder [10]. The definitions given above are generalizations of those of [9].

In general, the sum of two pseudomontone mappings with respect to $V$ is not pseudomonotone with respect to $V$, even for monotone mappings when $Y=Y_{0}=$ $Z=Z_{0}=l^{2}$, cf. $[5$, p. 101]. Some boundedness condition is needed.

Proposition 2.2. Let $\left(Y, Y_{0} ; Z, Z_{0}\right)$ be a complementary system and let $\left\{S_{t}: Y \rightarrow 2^{Z} ; t \in[0,1]\right\}$ and $\left\{T_{t}: Y \rightarrow 2^{Z} ; t \in[0,1]\right\}$ be two pseudomonotone bomotopies with respect to a dense subspace $V$ of $Y_{0}$. Suppose that for each bounded set $A$ in $V$,

$$
\bigcup\left\{S_{t}(y) ; y \in A \text { and } t \in[0,1]\right\}
$$

is bounded in $Z$. Then $\left\{S_{t}+T_{t}: Y \rightarrow 2^{Z} ; t \in[0,1]\right\}$ is a pseudomonotone bomotopy with respect to $V$.

Proof. The finite continuity of $S+T$ follows easily from the $\sigma(Z, V)$ compactness of $S_{t}(y)$ and $T_{t}(y)$ for each $t \in[0,1]$ and $y \in V$. Let $\left(t_{i}, y_{i}, z_{i}\right)$ be a net such that $z_{i} \in\left(S_{t_{i}}+T_{t_{i}}\right)\left(y_{i}\right), t_{i} \rightarrow t_{,} y_{i} \in V, y_{i}$ bounded, $y_{i} \rightarrow y \in Y$ for $\sigma\left(Y, Z_{0}\right), z_{i} \rightarrow z \in Z$ for $\sigma(Z, V)$ and

$$
\lim \sup \left\langle y_{i}, z_{i}\right\rangle \leq\langle y, z\rangle .
$$

We must show that $z \in\left(S_{i}+T_{t}\right)(y)$ and $\left\langle y_{i}, z_{i}\right\rangle \rightarrow\langle y, z\rangle$. Clearly, it suffices to 
prove the latter convergence for a subnet. Write $z_{i}=u_{i}+v_{i}$ with $u_{i} \in S_{t_{i}}\left(y_{i}\right)$ and $v_{i} \in T_{t_{i}}\left(y_{i}\right)$. Since $y_{i}$ remains bounded in $V, u_{i}$ remains bounded in $Z$, and thus, passing to a subnet, we can assume that $u_{i} \rightarrow u$ for $\sigma\left(Z, Y_{0}\right)$. We claim that

$$
\lim \sup \left\langle y_{i}, u_{i}\right\rangle \leq\langle y, u\rangle
$$

Indeed, if this is not true, then, for a subnet, $\left.\left\langle y_{i}, u_{i}\right\rangle \rightarrow \alpha\right\rangle\langle y, u\rangle$, and it follows from (2.1) that

$$
\lim \sup \left\langle y_{i}, v_{i}\right\rangle=\lim \sup \left\langle y_{i}, z_{i}-u_{i}\right\rangle\langle\langle y, z-u\rangle
$$

but $v_{i} \rightarrow z-u$ for $\sigma(Z, V)$, so that by the pseudomonotonicity of $\left\{T_{t}\right\},\left\langle y_{i}, v_{i}\right\rangle$ $\rightarrow\langle y, z-u\rangle$, which contradicts (2.3). Now (2.2) and the pseudomonotonicity of $\left\{S_{t}\right\}$ imply $u \in S_{t}(y)$ and $\left\langle y_{i}, u_{i}\right\rangle \rightarrow\langle y, u\rangle$. Replacing in (2.1), we obtain

$$
\lim \sup \left\langle y_{i}, v_{i}\right\rangle \leq\langle y, z-u\rangle,
$$

and consequently, by the pseudomonotonicity of $\left\{T_{t}\right\}, z-u \in T_{t}(y)$ and $\left\langle y_{i}, v_{i}\right\rangle$ $\rightarrow\langle y, z-u\rangle$. Q.E.D.

A one-parameter family of mappings $S_{t}$ from $Y$ into $2^{Z}, t \in[0,1]$, is said to be bounded on $V$ if it satisfies the boundedness assumption of Proposition 2.2. It is said to be strongly quasibounded on $V$ with respect to $\bar{y} \in V$ if for each $c_{1}, c_{2}>0$ there exists $k\left(c_{1}, c_{2}\right)>0$ such that whenever $z \in S_{t}(y)$ with $t \in[0,1], y \in V,\|y\| \leq c_{1}$ and $\langle y-\bar{y}, z\rangle \leq c_{2}$, then $\|z\| \leq k\left(c_{1}, c_{2}\right)$. Of course, "bounded" implies "strongly quasibounded", but the converse is not true, even for individual monotone mappings when $Y=Y_{0}=Z=Z_{0}=V=l^{2}$, cf. [11, Proposition 14] and [27, p. 305]. Here is another example of a different nature.

Example 2.3. Consider the complementary system $\left(L_{M}(\Omega), E_{M}(\Omega) ; L_{\bar{M}}(\Omega)\right.$, $E_{\bar{M}}(\Omega)$ ), where $M$ is assumed to have a continuous first derivative $p$, and define $S: D(S) \subset L_{M}(\Omega) \rightarrow L_{\bar{M}}(\Omega)$ by

$$
D(S)=\left\{u \in L_{M}(\Omega) ; p(u(x)) \in L_{\bar{M}}(\Omega)\right\}, \quad S u=p(u) .
$$

Clearly, $S$ is monotone, and it is easily verified that $E_{M}(\Omega) \subset D(S) \subset \mathcal{L}_{M}(\Omega)$. The argument of $\left[11\right.$, Proposition 14] shows that in a complementary system $\left(Y, Y_{0}\right.$; $\left.Z, Z_{0}\right)$ with $Y_{0}$ complete, a monotone mapping from $Y$ into $2^{Z}$ is strongly quasibounded on $Y_{0}$ with respect to $\bar{y} \in Y_{0}$ provided its domain contains some ball $B_{\epsilon}\left(\bar{y}, Y_{0}\right), \epsilon>0$. Hence $S$ is strongly quasibounded on $E_{M}(\Omega)$ with respect to any point of $E_{M}(\Omega)$. However, $S$ will be bounded on $E_{M}(\Omega)$ if and only if $E_{M}(\Omega)=L_{M}(\Omega)$. The "if" part follows from [24, p. 173]. To prove the "only if" part let $u \in L_{M}(\Omega)$ and define 


$$
u_{n}(x)= \begin{cases}u(x) & \text { if }|x| \leq n \text { and }|u(x)| \leq n, \\ 0 & \text { otherwise. }\end{cases}
$$

Since $u_{n}$ remains bounded in $E_{M}(\Omega), S u_{n}$ remains bounded in $L_{\bar{M}}(\Omega)$. Thus there exists $K>0$ such that

$$
K \geq \int_{Q} u_{n} p\left(u_{n}\right) d x=\int_{Q} M\left(u_{n}\right) d x+\int_{Q} \bar{M}\left(p\left(u_{n}\right)\right) d x \geq \int_{Q} M\left(u_{n}\right) d x
$$

for all $n$, and consequently, by Fatou's lemma, $\int_{Q} M(u) d x \leq K<+\infty$, i.e. $u \in$ $\mathfrak{L}_{M}(\Omega)$. Hence $L_{M}(\Omega) \subset \mathfrak{L}_{M}(\Omega)$, which implies $L_{M}(\Omega)=E_{M}(\Omega)$. In $\$ 4$ we will see that $S$ is pseudomontone with respect to any dense subspace $V$ of $E_{M}(\Omega)$.

The following proposition is closely related to Theorem 1 of Browder-Hess [11] where the notion of strong quasiboundedness was introduced.

Proposition 2.4. Let $\left(Y, Y_{0} ; Z, Z_{0}\right)$ be a complementary system and let $\left\{S_{i}: Y \rightarrow 2^{Z} ; t \in[0,1]\right\}$ and $\left\{T_{i}: Y \rightarrow 2^{Z} ; t \in[0,1]\right\}$ be two pseudomonotone bomotopies with respect to a dense subspace $V$ of $Y_{0}$. Suppose that $\left\{S_{t}\right\}$ is strongly quasibounded on $V$ with respect to some $\bar{y} \in V$, and that there exists $b: \mathbf{R} \rightarrow \mathbf{R}$ continuous such that whenever $z \in T_{t}(y)$ with $t \in[0,1]$ and $y \in V$, then $\langle y-y, z\rangle \geq$ $-b(\|y\|)\|y\|$. Then $\left\{S_{t}+T_{t}: Y \rightarrow 2^{Z} ; t \in[0,1]\right\}$ is a pseudomonotone bomotopy with respect to $V$.

Proof. With the notations of the proof of Proposition 2.2, we have, passing to a subnet if necessary,

$$
\left\langle y_{i}-\bar{y}, u_{i}\right\rangle=\left\langle y_{i}-\bar{y}, z_{i}\right\rangle-\left\langle y_{i}-\bar{y}, v_{i}\right\rangle \leq\langle y-\bar{y}, z\rangle+\epsilon+b\left(\left\|y_{i}\right\|\right)\left\|y_{i}\right\| \leq c,
$$

which implies, by the strong quasiboundedness of $\left\{S_{t}\right\}$, that $u_{i}$ remains bounded in $Z$. The proof can then be completed exactly as that of Proposition 2.2. Q.E.D.

If $\left\{S_{t}\right\}$ and $\left\{T_{t}\right\}$ are two sequentially pseudomonotone homotopies with respect to $V$ and if the boundedness assumptions of either Proposition 2.2 or 2.4 are satisfied, then $\left\{S_{t}+T_{t}\right\}$ is a sequentially pseudomonotone homotopy with respect to $V$ provided $Y_{0}$ is separable. The proofs are similar.

Remark 2.5. The sum of two mappings of type (M) with respect to $V$ is not necessarily of type (M) with respect to $V$, even when $Y=Y_{0}=Z=Z Z_{0}=V=l^{2}$ and both mappings are bounded on $V$, cf. [2, p. 128]. Note that a compact mapping $T$ (i.e. $D(T)=Y, T$ single-valued, continuous from $Y$ to $Z$ and the images of bounded sets in $Y$ are relatively compact in $Z$ ) may not be of type (M) with respect to any $V$ : consider $T: l^{2} \rightarrow l^{2}$ defined by $T x=(\|x\|, 0, \ldots)$. However a completely continuous mapping $T$ (i.e. $D(T)=Y, T$ single-valued and continuous on each bounded set of $Y$ from $\sigma\left(Y, Z_{0}\right)$ to the norm topology of $\left.Z\right)$ is pseudomonotone with respect to any $V$. 
We now turn to the study of the duality mapping. Let $\left(Y, Y_{0} ; Z, Z_{0}\right)$ be a complementary system. Let \|\|$_{Y}$ be a (equivalent) norm on $Y$ and let \|\|$_{Y_{0}}$, \|\|$_{Z}$ and \|\|$_{Z_{0}}$ be defined as in $\$ 1$. The corresponding (normalized) duality mapping $J: Y \rightarrow 2^{Z}$ is defined by

$$
J y=\left\{z \in Z ;\|z\|_{Z}=\|y\|_{Y} \text { and }\langle y, z\rangle=\|y\|_{Y}\|z\|_{Z}\right\} .
$$

The restriction of $J$ to $Y_{0}$ is the usual duality mapping from the normed space $Y_{0}$ into $2^{Y_{0}^{*}}$. Note that $J$ is bounded on $Y$.

Proposition 2.6. If \|\|$_{Y}$ is admissible, then $J: Y \rightarrow 2^{Z}$ is pseudomonotone with respect to any dense subspace $V$ of $Y_{0}$.

Proof. Let $V$ be a dense subspace of $Y_{0}$. The finite continuity of $J$ follows from the well-known properties of the usual duality mapping (cf. e.g. $[6, \S 7]$ ). Let $\left(y_{i}, z_{i}\right)$ be a net such that $z_{i} \in J y_{i}, y_{i} \in V, y_{i}$ bounded, $y_{i} \rightarrow y \in Y$ for $\sigma\left(Y, Z_{0}\right), z_{i} \rightarrow z \in Z$ for $\sigma(Z, V)$ and $\lim \sup \left\langle y_{i}, z_{i}\right\rangle \leq\langle y, z\rangle$. Since \|\|$_{Y}$ is admissible, we have

$$
\begin{aligned}
\|y\|_{Y}\|z\|_{Z} & \geq\langle y, z\rangle \geq \lim \sup \left\langle y_{i}, z_{i}\right\rangle \geq \lim \inf \left\langle y_{i}, z_{i}\right\rangle \\
& =\lim \inf \left\|y_{i}\right\|_{Y}\left\|z_{i}\right\|_{Z} \geq\|y\|_{Y}^{2} \text { and }\|z\|_{Z}^{2}
\end{aligned}
$$

because \|\|$_{Y}$ is $\sigma\left(Y, Z_{0}\right)$ 1.s.c. and \|\|$_{Z}$ is $\sigma(Z, V)$ 1.s.c. Consequently, $\|y\|_{Y}=\|z\|_{Z}$ and $\langle y, z\rangle=\|y\|_{Y}\|z\|_{Z}$, i.e. $z \in J y$. Moreover, $\left\langle y_{i}, z_{i}\right\rangle \rightarrow\langle y, z\rangle$ (and in addition $\left\|y_{i}\right\|_{Y} \rightarrow\|y\|_{Y}$ and $\left\|z_{i}\right\|_{Z} \rightarrow\|z\|_{Z}$ ). Q.E.D.

The following lemma is, in some technical sense, a substitute for the notion of mapping of type (S)+ (cf. [6], [9]) whose introduction in our general context seems useless.

Lemma 2.7. Let $\left(Y, Y_{0} ; Z, Z_{0}\right)$ be a complementary system and let $\left\{T_{t}: Y\right.$ $\left.\rightarrow 2^{Z} ; t \in[0,1]\right\}$ be a pseudomonotone bomotopy with respect to a dense subspace $V$ of $Y_{0}$. Let $J$ be the duality mapping corresponding to an admissible norm \|\|$_{Y}$ on $Y$. If a net $\left(t_{i}, y_{i}, z_{i}\right)$ satisfies $z_{i} \in\left(J+T_{t_{i}}\right)\left(y_{i}\right), t_{i} \rightarrow t, y_{i} \in V$, $y_{i}$ bounded, $y_{i} \rightarrow y \in Y$ for $\sigma\left(Y, Z_{0}\right), z_{i} \rightarrow z \in Z$ for $\sigma(Z, V)$ and $\lim \sup \left\langle y_{i}, z_{i}\right\rangle$ $\leq\langle y, z\rangle$, then $z \in\left(J+T_{j}\right)(y),\left\langle y_{i}, z_{i}\right\rangle \rightarrow\langle y, z\rangle$ and $\left\|y_{i}\right\|_{Y} \rightarrow\|y\|_{Y^{\bullet}}$

Proof. The first two assertions follow from Propositions 2.6 and 2.2, and it suffices to prove the last assertion for a subnet. Writing $z_{i}=u_{i}+v_{i}$ with $u_{i} \epsilon$ $J y_{i}$ and $v_{i} \in T_{t_{i}}\left(y_{i}\right)$, we obtain, as in the proof of Proposition 2.2, $u_{i} \rightarrow u$ for $\sigma(Z, V)$ and $\lim \sup \left\langle y_{i}, u_{i}\right\rangle \leq\langle y, u\rangle$. It follows, by the argument of the proof of Proposition 2.6, that $\left\|y_{i}\right\|_{Y} \rightarrow\|y\|_{Y}$. Q.E.D. 
3. Abstract existence theorems. This section contains our main existence theorems for functional equations involving mappings of monotone type in complementary systems. In the first theorem the mapping is assumed to be coercive, an assumption which is progressively weakened in the following theorems.

Theorem 3.1. Let $\left(Y, Y_{0} ; Z, Z_{0}\right)$ be a complementary system and let $T$ : $Y \rightarrow 2^{Z}$ be a mapping of type (M) with respect to a dense subspace $V$ of $Y_{0}$. Suppose that $T$ is coercive on $V$ with respect to some $\bar{y} \in V$, i.e., that

$$
\inf \left\{\langle y-\bar{y}, z\rangle\|y\|^{-1} ; z \in T y\right\} \rightarrow+\infty \text { as }\|y\| \rightarrow \infty, y \in V .
$$

Then the range $R(T)$ of $T$ contains $Z_{0^{*}}$.

Proof. Let $\mathcal{F}$ be the directed set of all finite-dimensional subspaces $F$ of $V$ containing $\bar{y}$ ordered by inclusion. For each $F \in \mathcal{F}$, denote by $j_{F}$ the injection of $F$ into $V$, by $j_{F}^{*}$ the dual projection of $Z$ onto $F^{*}$ and by $T_{F}=j_{F}^{*} T j_{F}$ : $F \rightarrow 2^{F^{*}}$ the Galerkin approximant of $T$. Clearly, the mapping $T_{F}$ is upper semicontinuous, takes values in the nonempty compact convex subsets of $F^{*}$ and is coercive on $F$. This implies, by a standard argument (cf. e.g. $[7$, p. 10]) based on the multivalued version of the Brouwer fixed point theorem $[6, \$ 6]$, that $R\left(T_{F}\right)=F^{*}$. In particular, given $z \in Z_{0}$, there exists $y_{F} \in F$ and $z_{F} \in T y_{F}$ such that $j_{F}^{*} z_{F}=j_{F}^{*} z$. It follows from the coercivity of $T$ that $y_{F}$ remains bounded in $V$ as $F \in \mathcal{F}$. Hence, passing to a subnet, we can assume that $y_{F} \rightarrow$ $y \in Y$ for $\sigma\left(Y, Z_{0}\right)$. We have $z_{F} \rightarrow z$ for $\sigma(Z, V)$ because $\left\langle u, z_{F}\right\rangle=\langle u, z\rangle$ as soon as $F$ contains $u$. Moreover, since $z \in Z_{0}$,

$$
\left.' y_{F}, z_{F}\right\rangle=\left\langle y_{F}, z\right\rangle \rightarrow\langle y, z\rangle \text {. }
$$

Consequently, by the type (M) property of $T, z \in T y$. Q.E.D.

If $T$ is sequentially of type (M) with respect to any dense subspace $V$ of a dense subspace $V^{\prime}$ of $Y_{0}$ and if $T$ is coercive on $V^{\prime}$ with respect to $\bar{y} \in V^{\prime}$, then $R(T) \supset Z_{0}$ provided $Y_{0}$ and $Z_{0}$ are separable. The proof is similar and is obtained by starting with an increasing sequence of finite-dimensional subspaces of $V^{\prime}$ containing $\bar{y}$, whose union $V$ is dense in $V^{\prime}$.

Example 3.2. Let $T$ be a maximal monotone mapping from a Banach space $X$ into $2^{X^{*}}$ and consider the mapping $T_{1}: X^{* *} \rightarrow 2^{X^{*}}$ whose graph is given by gr $T_{1}=\left\{\left(x^{* *}, x^{*}\right)\right.$; there exists a net $\left(x_{i}, x_{i}^{*}\right) \in \mathrm{gr} T$ with

$$
\begin{aligned}
x_{i} \text { bounded in } X, x_{i} \rightarrow & x^{* *} \text { for } \sigma\left(X^{* *}, X^{*}\right) \\
& \text { and } x_{i}^{*} \rightarrow x^{*} \text { in norm\}. }
\end{aligned}
$$

Assume that $T_{1}$ is maximal monotone from $X^{* *}$ into $2^{X^{*}}$. (This is the case for 
instance if $T$ is the subdifferential of a convex function [13], [16], or the monotone operator associated with a saddle function [18].) Then, by an easy generalization of the arguments of [16], $R\left(T_{1}\right)=X^{*}$ when $T$ is coercive on its domain

$$
D(T)=\{x \in X ; T x \text { nonempty }\}
$$

with respect to a point of its domain. Under the additional assumptions that $D(T)$ is a dense subspace of $X$ and $T$ is finitely continuous from $D(T)$ to the $\sigma\left(X^{*}, D(T)\right)$ topology of $X^{*}$, this result also follows from Theorem 3.1. Indeed, one can then show that in the complementary system $\left(X^{* *}, X ; X^{*}, X^{*}\right), T_{1}$ is pseudomonotone with respect to $D(T)$.

Example 3.3. Let $T$ be a maximal monotone mapping from a Banach space $X$ into $2^{X^{*}}$ and define $T_{2}: X^{* *} \rightarrow 2^{X^{*}}$ by

gr $T_{2}=\left\{\left(x^{* *}, x^{*}\right) ;\right.$ there exists a net $\left(x_{i}, x_{i}^{*}\right) \in \mathrm{gr} T$ with

$$
\begin{aligned}
& x_{i} \text { bounded in } X, x_{i} \rightarrow x^{* *} \text { for } \sigma\left(X^{* *}, X^{*}\right), \\
& \left.x_{i}^{*} \rightarrow x^{*} \text { for } \sigma\left(X^{*}, D(T)\right) \text { and } \lim \sup \left\langle x_{i}, x_{i}^{*}\right\rangle \leq\left\langle x^{* *}, x^{*}\right)\right\}
\end{aligned}
$$

If $D(T)$ is a dense subspace of $X$ and if $T$ is finitely continuous from $D(T)$ to the $\sigma\left(X^{*}, D(T)\right)$ topology of $X^{*}$, then, in the complementary system $\left(X^{* *}, X\right.$; $\left.X^{*}, X^{*}\right), T_{2}$ is of type (M) with respect to $D(T)$. Consequently $R\left(T_{2}\right)=X^{*}$ when $T$ is coercive on $D(T)$ with respect to a point of $D(T)$. This result essentially contains the existence theorem of Donaldson [12].

Remark 3.4. It is not known whether the range of a maximal monotone mapping $T: X^{*} \rightarrow 2^{X}, X$ a Banach space, $T$ coercive on $D(T)$ with respect to a point of $D(T)$, is all of $X$. This is true if $D(T)=X^{*}$ and $T$ is single-valued and finitely continuous from $X^{*}$ to the $\sigma\left(X, X^{*}\right.$ ) topology of $X$ (cf. [4]; however it is not clear whether a mapping satisfying all those conditions exists, unless $X$ is reflexive) or more generally (cf. [16, p. 387]), using the arguments of [32, p. 405-406], if the norm closure of $R(T)$ is convex. By means of the method of Theorem 3.1, one can give another extension of the result of [4]: $R(T)=X$ if $D(T)$ is a $\sigma\left(X^{*}, X\right)$ dense subspace of $X^{*}$ and $T$ is finitely continuous from $D(T)$ to the $\sigma(X, D(T))$ topology of $X$.

Theorem 3.5. Let $\left(Y, Y_{0} ; Z, Z_{0}\right)$ be a complementary system and let $T$ : $D(T) \subset Y \rightarrow 2^{Z}$ be a pseudomonotone mapping with respect to a dense subspace $V$ of $Y_{0}$. Suppose that for some $\bar{y} \in V$,

$$
\inf \left\{\langle y-\bar{y}, z\rangle\|y\|^{-1}+\|z\| ; z \in T y\right\} \rightarrow+\infty \quad \text { as }\|y\| \rightarrow \infty, y \in D(T),
$$

and that there exists $b: \mathbf{R}^{+} \rightarrow \mathbf{R}^{+}$continuous sucb that $\inf \{\langle y-\bar{y}, z\rangle ; z \in T y\} \geq$ 
$-b(\|y\|)$ for $y \in V$ witb $\|y\|$ sufficiently large. Suppose also that $Y$ admits an equivalent admissible norm \|\|$_{Y^{*}}$ Then $R(T) \supset Z_{0^{*}}$

Proof. For simplicity we will assume that $T$ is single-valued and that the restriction of \|\|$_{Y}$ to $Y_{0}$ is Gâteaux differentiable. The same arguments carry over immediately to the general case, using the degree theory for multivalued mappings (cf. [20]).

Since the assumptions and the conclusion are invariant by adding to $T$ a fixed element of $Z_{0}$ or by translating $T$ by a fixed element of $V$, it suffices to show that $0 \in R(T)$ and we can assume that $\bar{y}=0$. Endow $Y$ with \|\|$_{Y^{\circ}}$ Choose $R>0$ so large that

$$
\langle y, T y\rangle\|y\|_{Y}^{-1}+\|T y\|_{Z}>0
$$

for all $y \in S_{R}(0, Y) \cap D(T)$, and that for some $H>0$,

$$
\langle y, T y\rangle \geq-H
$$

for all $y$ on the sphere $S_{R}(0, V)$. Let $J: Y \rightarrow 2^{Z}$ be the duality mapping corresponding to \|\|$_{Y}$. Note that $J$ is single-valued on $Y_{0^{*}}$. For $t \in[0,1]$, define $T_{t}=(1-t) T+t J$. Let $\mathcal{F}$ be the directed set of all finite-dimensional subspaces $F$ of $V$ and denote by $T_{F}, J_{F}, T_{t, F}$ the Galerkin approximants of $T, J, T_{t}$ respectively. Clearly, $T_{t, F}=(1-t) T_{F}+t J_{F}$.

If there exists $F_{0} \in \dot{\mathcal{F}}$ such that $T_{t, F}(y) \neq 0$ for all $F \supset F_{0}, t \in[0,1]$ and $y \in S_{R}(0, F)$, then, by a finite-dimensional degree argument (cf. e.g., [9, Theorem 8]), we can find for each $F \supset F_{0}$ an element $y_{F} \in B_{R}(0, F)$ satisfying $T_{F}\left(y_{F}\right)=0$. It is then easy to go to the limit using the pseudomonotonicity of $T$ and to obtain $0 \in R(T)$.

In the contrary case, there exist a cofinal subset of $\mathcal{F}$, still denoted by $\{F\}$ for simplicity, $t_{F} \in[0,1]$ and $y_{F} \in S_{R}(0, F)$ such that $T_{t_{F}, F}\left(y_{F}\right)=0$. We can assume that $t_{F} \rightarrow t \in[0,1]$ and $y_{F} \rightarrow y \in Y$ for $\sigma\left(Y, Z_{0}\right)$. Three cases must be distinguished: $t=0,0<t<1$ and $t=1$. If $t=0$, then $T y_{F} \rightarrow 0$, for $\sigma(Z, V)$ because

$$
\begin{aligned}
\left\langle u, T y_{F}\right\rangle & =\left(1-t_{F}\right)^{-1}\left\langle u, T_{t_{F}}\left(y_{F}\right)\right\rangle-t_{F}\left(1-t_{F}\right)^{-1}\left\langle u, J y_{F}\right\rangle \\
& =-t_{F}\left(1-t_{F}\right)^{-1}\left\langle u, J y_{F}\right\rangle
\end{aligned}
$$

as soon as $F$ contains $u$. Moreover,

$$
\left\langle y_{F}, T y_{F}\right\rangle=-t_{F}\left(1-t_{F}\right)^{-1}\left\|y_{F}\right\|_{Y}^{2} \rightarrow 0 \text {. }
$$

Consequently, by the pseudomonotonicity of $T, T y=0$, and thus $0 \in R(T)$. If $0<t<1$, then $T_{t} y_{F} \rightarrow 0$ for $\sigma(Z, V)$ because, using (3.3), 


$$
\begin{aligned}
\left\langle u, T_{t}\left(y_{F}\right)\right\rangle & =\left\langle u, T_{t_{F}}\left(y_{F}\right)\right\rangle+\left(t_{F}-t\right)\left(\left\langle u, T y_{F}\right\rangle-\left\langle u, J y_{F}\right\rangle\right) \\
& =\left(t_{F}-t\right)\left(-t_{F}\left(1-t_{F}\right)^{-1}-1\right)\left\langle u, J y_{F}\right\rangle
\end{aligned}
$$

as soon as $F$ contains $u$. Moreover

$$
\left\langle y_{F}, T_{t}\left(y_{F}\right)\right\rangle=\left(t_{F}-t\right)\left(-t_{F}\left(1-t_{F}\right)^{-1}-1\right)\left\|y_{F}\right\|^{2} \rightarrow 0 .
$$

Consequently, by the pseudomonotonicity of $T_{t}$ (cf. Proposition 2.2), $0 \in T_{t}(y)$. In addition, by Lemma 2.7, $\left\|y_{F}\right\|_{Y} \rightarrow\|y\|_{Y}$, so that $y \in S_{R}(0, Y)$. But this contradicts (3:1) because it follows from $0=(1-t) T y+t z$ with $z \in J y$ that

$$
\langle y, T y\rangle\|y\|_{Y}^{-1}+\|T y\|_{Z}=-t(1-t)^{-1} R+t(1-t)^{-1} R=0 .
$$

Finally, if $t=1$, then

$$
\left\langle y_{F}, T y_{F}\right\rangle=-t_{F}\left(1-t_{F}\right)^{-1}\left\|y_{F}\right\|_{Y}^{2} \rightarrow-\infty,
$$

which contradicts (3.2). Q.E.D.

Remark 3.6. The assumption involving $b$ is automatically satisfied if $T$ is monotone. It can also be replaced by the assumption that $T$ is strongly quasibounded on $V$ with respect to $\bar{y}$.

In the sequential version of Theorem 3.5, one requires that $T$ be sequentially pseudomonotone with respect to any dense subspace $V$ of a dense subspace $V^{\prime}$ of $Y_{0}$, that $\bar{y} \in V^{\prime}$, that the inequality $\inf \{\langle y-\bar{y}, z\rangle ; z \in T y\} \geq-b(\|y\|)$ holds for $y \in V^{\prime}$ with $\|y\|$ sufficiently large, and that $Y_{0}$ and $Z_{0}$ be separable.

As a specialization of Theorem 3.5 , we have

Corollary 3.7. Let $\left(Y, Y_{0} ; Z, Z_{0}\right)$ be a complementary system and let $T$ : $D(T) \subset Y \rightarrow 2^{Z}$ be a pseudomonotone mapping with respect to a dense subspace $V$ of $Y_{0}$. Suppose that there exists $k>0$ such that for some $\bar{y} \in V$,

$$
\inf \left\{\langle y-\bar{y}, z\rangle\|y\|^{-1} ; z \in T y\right\} \geq-k
$$

for $y \in D(T)$ with $\|y\|$ sufficiently large, and that $T^{-1}: Z \rightarrow 2^{Y}$ is bounded on $R(T)$. Suppose also that $Y$ admits an equivalent admissible norm \|\|$_{Y^{*}}$ Then $R(T) \supset Z_{0}$.

Theorems 3.1 and 3.5 generalize results of Brézis [2], Browder [3], [7], [9] and Browder-Hess [11]. The very weak coercivity condition in Theorem 3.5 was introduced in [0] as a weakening of the so-called subcoercivity condition of Corollary 3.7 considered in [11]. These asymptotic conditions imply the existence of a global a priori bound, i.e. that $T^{-1}: Z \rightarrow 2^{Y}$ is bounded on $R(T)$. (Note that the example $T: \mathbf{R} \rightarrow \mathbf{R :} x \rightarrow x^{2}$ shows that a global a priori bound is not sufficient in general to get surjectivity.) In the next two theorems, only a local 
a priori bound is needed. However some additional structural condition must be imposed on the mapping $T$, either some oddness condition or some stronger monotonicity condition.

Theorem 3.8. Let $\left(Y, Y_{0} ; Z, Z_{0}\right)$ be a complementary system and let $\left\{T_{t}: D\left(T_{t}\right) \subset Y \rightarrow 2^{Z} ; t \in[0,1]\right\}$ be a pseudomonotone bomotopy with respect to a dense subspace $V$ of $Y_{0}$. Suppose that $T_{1}$ is odd on $V$ outside some ball of $V$. Suppose that eacb $z \in Z_{0}$ bas a (norm) neigbbourbood $\pi$ in $Z$ such that

$$
\bigcup\left\{T_{t}^{-1}(z) ; z \in \Re \text { and } t \in[0,1]\right\}
$$

is bounded in $Y$. Suppose also that $Y$ admits an equivalent admissible norm \|\|$_{Y^{*}}$ Then $R\left(T_{t}\right) \supset Z_{0}$ for each $t \in[0,1]$.

Proof. For simplicity we will assume that $T$ is single-valued and that the restriction of \|\|$_{Y}$ to $Y_{0}$ is Gâteaux differentiable. The same arguments carry over immediately to the general case, using the Borsuk-Ulam theorem for multivalued mappings (cf. [21]).

It suffices to show that $R\left(T_{0}\right) \supset Z_{0^{\circ}}$. Let $z \in Z_{0^{\circ}}$. Endow $Y$ with \|\|$_{Y^{*}}$ By a compactness argument, there exist a neighbourhood $\gamma$ in $Z$ of the segment $[0, z]$ and $R>0$ such that $T_{t}(y) \notin \pi$ for all $t \in[0,1]$ and $y \in S_{R}(0, Y) \cap D\left(T_{t}\right)$. Taking $R$ larger if necessary, we can assume that $T_{1}(-y)=-T_{1}(y)$ for $y \in V$ with $y \notin B_{R}(0, V)$. Let $J: Y \rightarrow 2^{Z}$ be the duality mapping corresponding to \|\|$_{Y}$. Define $T_{t, \epsilon}=T_{t}+\epsilon J$ and choose $\epsilon_{0}>0$ such that $T_{t, \epsilon}(y) \notin[0, z]$ for all $t \in[0,1], 0 \leq \epsilon \leq \epsilon_{0}$ and $y \in S_{R}(0, Y) \cap D\left(T_{t}\right)$. Let $\mathcal{F}$ be the directed set of all finite-dimensional subspaces of $V$ and denote by $T_{t, F}, J_{F}, T_{t, \epsilon, F}$ the Galerkin approximants of $T_{t}, J, T_{t, \epsilon}$ respectively. Clearly, $T_{t, \epsilon, F}=T_{t, F}+\epsilon J_{F}$ and $T_{1, \epsilon, F}$ is odd on $F$ outside $B_{R}(0, F)$.

Suppose first that for each $\epsilon$ with $0<\epsilon \leq \epsilon_{0}$ and each $F \in \mathcal{F}$ there exists $F^{\prime}=F^{\prime}(\epsilon, F)$ with $F^{\prime} \supset F$ such that $T_{t, \epsilon, F^{\prime}}(y) \neq \xi_{j}^{*},(z)$ for all $t \in[0,1], y \epsilon$ $S_{R}\left(0, F^{\prime}\right)$ and $\xi \in[0,1]$. Then, by a finite-dimensional degree argument (cf. e.g. $\left[\vartheta\right.$, Theorem 9]) based on the Borsuk-Ulam theorem, we can find $y_{\epsilon, F^{\prime}} \in B_{R}\left(0, F^{\prime}\right)$ satisfying $T_{0, \epsilon, F^{\prime}}\left(y_{\epsilon, F^{\prime}}\right)=j_{F}^{*},(z)$. Consider now the cofinal subset of $\mathscr{F}_{\mathfrak{F}}$ consisting of those $F^{\prime}(\epsilon, F)^{\prime}$ s as $\epsilon \rightarrow 0$ and $F \in \mathcal{F}$. We can assume that $y_{\epsilon, F^{\prime}} \rightarrow y \in Y$ for $\sigma\left(Y, Z_{0}\right)$. We have $T_{0}\left(y_{\epsilon, F^{\prime}}\right) \rightarrow z$ for $\sigma(Z, V)$ because

$$
\begin{aligned}
\left\langle u, T_{0}\left(y_{\epsilon, F^{\prime}}\right)\right\rangle & =\left\langle u, T_{0, \epsilon}\left(y_{\epsilon, F^{\prime}}\right)\right\rangle-\epsilon\left\langle u, J y_{\epsilon, F^{\prime}}\right\rangle \\
& =\langle u, z\rangle-\epsilon\left\langle u, J y_{\epsilon, F^{\prime}}\right\rangle
\end{aligned}
$$

as soon as $F^{\prime}$ contains $u$. Moreover, since $z \in Z_{0}$, 


$$
\left\langle y_{\epsilon, F^{\prime}}, T_{0}\left(y_{\epsilon, F^{\prime}}\right)\right\rangle=\left\langle y_{\epsilon, F^{\prime}}, z\right\rangle-\epsilon\left\|y_{\epsilon, F^{\prime}}\right\|_{Y}^{2} \rightarrow\langle y, z\rangle .
$$

Consequently, by the pseudomonotonicity of $T_{0}, z=T_{0}(y)$. Thus $z \in R(T)$.

In the contrary case, there exist $\epsilon$ with $0<\epsilon \leq \epsilon_{0}$, a cofinal subset of $\mathcal{F}$, still denoted by $\{F\}$ for simplicity, $t_{F} \in[0,1], y_{F} \in S_{R}(0, F)$ and $\xi_{F} \in[0,1]$ such that $T_{t_{F}, \epsilon, F}\left(y_{F}\right)=\xi_{F} j_{F}^{*}(z)$. We can assume that $t_{F} \rightarrow t \in[0,1], \xi_{F} \rightarrow \xi$ $\epsilon[0,1]$, and $y_{F} \rightarrow y \in Y$ for $\sigma\left(Y, Z_{0}\right)$. We have $T_{t_{F}, \epsilon}\left(y_{F}\right) \rightarrow \xi z$ for $\sigma(Z, V)$ because

$$
\left\langle u, T_{t_{F}, \epsilon}\left(y_{F}\right)\right\rangle=\left\langle u, \xi_{F} z\right\rangle
$$

as soon as $F$ contains $u$. Moreover, since $z \in Z_{0}$,

$$
\left\langle y_{F}, T_{t_{F}, \epsilon}\left(y_{F}\right)\right\rangle=\left\langle y_{F}, \xi_{F} z\right\rangle \rightarrow\langle y, \xi z\rangle \text {. }
$$

Consequently, since $\left\{T_{t, \epsilon}: Y \rightarrow 2^{Z} ; t \in[0,1]\right\}$ is a pseudomonotone homotopy with respect to $V$ (cf. Proposition 2.2), $\xi z=T_{t, \epsilon}(y)$. In addition, by Lemma 2.7, $\left\|y_{F}\right\|_{Y} \rightarrow\|y\|_{Y}$, so that $y \in S_{R}(0, Y)$. But this contradicts the definition of $\epsilon_{0}$. Q.E.D.

As a specialization of Theorem 3.8, we have

Corollary 3.9. Let $\left(Y, Y_{0} ; Z, Z_{0}\right)$ be a complementary system and let $T$ : $D(T) \subset Y \rightarrow 2^{Z}$ be a pseudomonotone mapping with respect to a dense subspace $V$ of $Y_{0}$. Suppose that $T$ is odd on $V$ outside some ball of $V$. Suppose that each $z \in Z_{0}$ bas a (norm) neigbbourbood in $Z$ whose image by $T^{-1}$ is bounded in $Y$. Suppose also that $Y$ admits an equivalent admissible norm \|\|$_{Y \cdot}$. Then $R(T) \supset Z_{0^{*}}$

Theorem 3.10. Let $\left(Y, Y_{0} ; Z, Z_{0}\right)$ be a complementary system and let $T$ : $Y \rightarrow 2^{Z}$ be a pseudomonotone mapping with respect to a dense subspace $V$ of $Y_{0}$. Suppose that $T$ is monotone and that its graph is closed in the following sense: if $\left(y_{i}, z_{i}\right) \in \mathrm{gr} T, y_{i} \rightarrow y \in Y$ for $\sigma\left(Y, Z_{0}\right), y_{i}$ bounded, $z_{i} \rightarrow z \in Z_{0}$ in norm, then $(y, z) \in \mathrm{gr} T$. Suppose that each $z \in Z_{0}$ bas a (norm) neighbourbood in $Z$ whose image by $T^{-1}$ is bounded in $Y$. Suppose also that $Y$ admits an equivalent admissible norm \|\|$_{Y^{*}}$. Finally suppose that $T(V)$ meets $Z_{0^{*}}$ Then $R(T) \supset Z_{0^{*}}$

Proof. Since the assumptions and the conclusion are invariant by translating $T$ by a fixed element of $V$, we can assume that $T(0)$ meets $Z_{0}$. Let $J$ be the duality mapping corresponding to \|\|$_{Y}$. Define $T_{\epsilon}=T+\epsilon J, \epsilon>0$. By Proposition 2.2, the monotonicity of $T$ and Theorem 3.1, $R\left(T_{\epsilon}\right) \supset Z_{0}$ for each $\epsilon>0$. Moreover, since $T_{\epsilon}^{-1}$ is bounded on its domain, the relations

$$
\begin{aligned}
\left\langle y-y^{\prime}, z-z^{\prime}\right\rangle & =\left\langle y-y^{\prime}, u-u^{\prime}\right\rangle+\left\langle y-y^{\prime}, v-v^{\prime}\right\rangle \\
& \geq \epsilon\left\langle y-y^{\prime}, v-v^{\prime}\right\rangle \geq \epsilon\left(\|y\|_{Y}-\left\|y^{\prime}\right\|_{Y}\right)^{2},
\end{aligned}
$$


where $z=u+v$ with $u \in T y$ and $v \in J y$ and similarly for $z^{\prime}$, show that the application $z \mapsto\left\|T_{\epsilon}^{-1}(z)\right\|_{Y}$ is single-valued and continuous on $R\left(T_{\epsilon}\right)$ for the norm of $Z$.

Let $z \in Z_{0}$ and take $y_{\epsilon} \in T_{\epsilon}^{-1}(z)_{\text {. We }}$ will show that $y_{\epsilon}$ remains bounded in $Y$ as $\epsilon \downarrow 0$. Then, taking a subnet such that $y_{\epsilon} \rightarrow y \in Y$ for $\sigma\left(Y, Z_{0}\right)$ and writing $z=$ $u_{\epsilon}+\epsilon v_{\epsilon}$ with $u_{\epsilon} \in T y_{\epsilon}$ and $v_{\epsilon} \in J y_{\epsilon}$, we obtain $u_{\epsilon} \rightarrow z$ in norm, so that, by the closedness of the graph of $T, z \in T y$. To show that $y_{\epsilon}$ remains bounded in $Y$ as $\epsilon \downarrow 0$, take $z_{0} \in T(0) \cap Z_{0}$. By a compactness argument, there exists $\delta>0$ such that

$$
T^{-1}\left\{u \in Z ; \operatorname{dist}\left(u ;\left[z, z_{1}\right]\right)<\delta\right\} \equiv T^{-1} \pi
$$

is bounded in $Y$, say $\left\|T^{-1}(u)\right\|_{Y} \leq K$ for all $u \in \pi$. Choose $\epsilon_{0}$ with $0<\epsilon_{0}<$ $\delta / 2 K$. We claim that $\left\|T_{\epsilon}^{-1}(u)\right\|_{Y} \leq 2 K$ for all $u \in\left[z, z_{1}\right]$ and $0<\epsilon<\epsilon_{0}$. Indeed, if this is not true, then for some $\epsilon$ with $0<\epsilon<\epsilon_{0}$ and some $u \in\left[z, z_{1}\right]$, $\left\|T_{\epsilon}^{-1}(u)\right\|_{Y}>2 K$. Since $\left\|T_{\epsilon}^{-1}\left(z_{1}\right)\right\|_{Y}=0$ and $\left\|T_{\epsilon}^{-1}(\cdot)\right\|_{Y}$ is continuous on $R\left(T_{\epsilon}\right)$ $\zeta Z_{0}$, there exists $z_{2} \epsilon\left[z_{,} z_{1}\right]$ with $\left\|T_{\epsilon}^{-1}\left(z_{2}\right)\right\|_{Y}=2 K$. Write $z_{2}=u_{2}+\epsilon v_{2}$ with $u_{2} \in T y_{2}$ and $v_{2} \in J y_{2}$. We have $\left\|y_{2}\right\|_{Y}=2 K$. On the other hand, the distance from $u_{2}$ to $z_{2} \in\left[z, z_{1}\right]$ is less than $\left\|\epsilon v_{2}\right\|_{Z}=\epsilon 2 K<\delta$. Thus $u_{2} \in \Re$ and consequently $y_{2} \in T^{-1}\left(u_{2}\right)$ must satisfy $\left\|y_{2}\right\|_{Y} \leq K$, a contradiction. Q.E.D.

Remark 3.11. If $T: Y \rightarrow 2^{Z}$ is strictly monotone (i.e. $\left\langle y_{1}-y_{2}, z_{1}-z_{2}\right\rangle>0$ for $\left(y_{1}, z_{1}\right)$ and $\left(y_{2}, z_{2}\right)$ in $\mathrm{gr} T$ with $\left.y_{1} \neq y_{2}\right)$, then the equation $z \in T y$ with $z \in Z$ has at most one solution $y \in D(T)$.

Remark 3.12. In the reflexive situation $Y=Y_{0}, Z=Z_{0}$, if $T: Y \rightarrow 2^{Z}$ is monotone and pseudomonotone with respect to a dense subspace $V$ of $Y_{0}$, then $T$ is maximal monotone. Indeed, endow $Y$ with a strictly convex equivalent norm (cf. [26]); it follows from Proposition 2.2 and Theorem 3.1 that $R(J+T)=Z$, which implies that $T$ is maximal monotone (cf. [33, p. 78]).

When $Y_{0}$ and $Z_{0}$ are separable, Theorems 3.8 and 3.10 have sequential versions: one requires in Theorem 3.8 that $\left\{T_{t}: D\left(T_{t}\right) \subset Y \rightarrow 2^{Z} ; t \in[0,1]\right\}$ be sequentially pseudomonotone with respect to any dense subspace $V$ of a dense subspace $V^{\prime}$ of $Y_{0}$ and that $T_{1}$ be odd on $V^{\prime}$ outside some ball of $V^{\prime}$, and in Theorem 3.10 that $T$ be sequentially pseudomonotone with respect to any dense subspace $V$ of a dense subspace $V^{\prime}$ of $Y_{0}$, that its graph be sequentially closed, and that $T\left(V^{\prime}\right)$ meet $Z_{0^{\circ}}$

Theorem 3.8 generalizes and sharpens results of Browder [8], [0] where a global a priori bound is required. Theorem 3.10 is a partial extension of Rockafellar's result [32] that a maximal monotone mapping $T$ from a reflexive Banach space $X$ into $2^{X^{*}}$ is onto if (and only if) each point of $X^{*}$ has a neighbourhood whose image by $T^{-1}$ is bounded in $X$.

The weakening of the usual asymptotic condition of coercivity is crucial for the applications, as is seen from the following example. 
Example 3.13. Consider the mapping $S: D(S) \subset L_{M}(\Omega) \rightarrow L_{\bar{M}}(\Omega)$ of Example 2.3, where $M$ and $\bar{M}$ are assumed to have continuous first derivatives $p$ and $\bar{p}$ respectively. Then $S$ is coercive on $E_{M}(\Omega)$ if and only if $E_{\bar{M}}(\Omega)=L_{\bar{M}}(\Omega)$. The "if" part follows from the inequality

$$
p(u) u=M(u)+\bar{M}(p(u)) \geq M(u), \quad u \in \mathbf{R},
$$

and Lemma 3.14 below. To verify the "only if" part, note that if $S$ is coercive on $E_{M}(\Omega)$, then $S^{-1}$ is bounded on the dense subspace of $E_{\bar{M}}(\Omega)$ consisting of all bounded functions with compact support in $\bar{\Omega}$, and thus, by the discussion in Example 2.3, $E_{\bar{M}}(\Omega)=L_{\bar{M}}(\Omega)$. Of course, $E_{M}(\Omega) \neq L_{M}(\Omega)$ and $E_{\bar{M}}(\Omega) \neq L_{\bar{M}}(\Omega)$ can happen simultaneously (cf. e.g. $[24$, p. 28]), in which case $S$ is not everywhere defined on $L_{M}(\Omega)$, nor bounded or coercive on $E_{M}(\Omega)$, and $S^{-1}$ is not bounded on $E_{\bar{M}}(\Omega)$. We will see in $\S 4$ that each point of $E_{\bar{M}}(\Omega)$ has a neighbourhood in $L_{\bar{M}}(\Omega)$ whose image by $S^{-1}$ is bounded in $L_{\bar{M}}(\Omega)$, so that the assumptions of Corollary 3.9 and Theorem 3.10 are satisfied by $S$. The present discussion also shows that the range of $T$ in Corollary 3.9 and Theorem 3.10 is not necessarily all of $Z$.

Lemma 3.14. Let $\Omega$ be an open subset of $\mathbf{R}^{n}$ and let $M$ be a $N$-function. If $L_{\bar{M}}(\Omega)=E_{\bar{M}}(\Omega)$, then

$$
\|u\|_{(M)}^{-1} \int_{\Omega} M(u) d x \rightarrow+\infty
$$

as $\|u\|_{(M)} \rightarrow+\infty, u \in L_{M}(\Omega)$.

Proof. First assume that $\Omega$ has infinite measure. Then $\bar{M}$ has the $\Delta_{2}$ property for all values of $t$ : there exists $k$ such that $\bar{M}(2 t) \leq k \bar{M}(t)$ for $t \in \mathbf{R}$. Necessarily, $k>2$. Defining a function $f:[1,+\infty[\rightarrow[k,+\infty[$ by

$$
f(r)=r\left((1-\lambda) k^{n+1}+\lambda k^{n+2}\right) \text { if } r \in\left[2^{n}, 2^{n+1}\right] \text { and } r=(1-\lambda) 2^{n}+\lambda 2^{n+1} \text {, }
$$

we obtain $\bar{M}(r t) \leq f(r) \bar{M}(t)$ for $t \in \mathbf{R}$ and $r \geq 1$, i.e. $M\left(f(r) r^{-1} t\right) \geq f(r) M(t)$ for $t \in \mathbf{R}$ and $r \geq 1$. Since $f(r) r^{-1}$ strictly increases from $k$ to $+\infty$ as $r \in[1,+\infty[$, its reciprocal function $g(s)$ is well defined and strictly increases from 1 to $+\infty$ as $s \in[k+\infty[$, and we have

$$
M(s t) \geq s g(s) M(t)
$$

for $t \in \mathbf{R}$ and $s \geq k$. Now take $u \in L_{M}(\Omega)$ with $\|u\|_{(M)}>k$. If $\epsilon>0$ satisfies $\|u\|_{(M)}-\epsilon>k$, then 


$$
\begin{aligned}
\int_{\Omega} M(u) d x & \geq\left(\|u\|_{(M)}-\epsilon\right) g\left(\|u\|_{(M)}-\epsilon\right) \int_{\Omega} M\left(u\left(\|u\|_{(M)}-\epsilon\right)^{-1}\right) d x \\
& \geq\left(\|u\|_{(M)}-\epsilon\right) g\left(\|u\|_{(M)}-\epsilon\right)
\end{aligned}
$$

by definition of the Luxemburg norm \|\|$_{(M)}$, so that

$$
\int_{\Omega} M(u) d x \geq\|u\|_{(M)} g\left(\|u\|_{(M)}\right),
$$

which proves the lemma when $\Omega$ has infinite measure.

If $\Omega$ has finite measure, then $\bar{M}$ has the $\Delta_{2}$ pioperty for large values of $t$, and thus (3.4) holds only for $t \geq t_{0}$ and $s \geq k$. For $u \in L_{M}(\Omega)$, denote by $\Omega_{u}$ the subset of $\Omega$ where $|u(x)| \geq t_{0}$. As above we obtain

$$
\int_{\mathbf{\Omega}_{u}} M(u) d x \geq\|u\|_{(M), \Omega_{u}} g\left(\|u\|_{(M), \Omega_{u}}\right)
$$

provided $\|u\|_{(M), \Omega_{u}}>k$, where $\|u\|_{(M), \Omega_{u}}$ denotes the Luxemburg norm of $u$ in $L_{M}\left(\Omega_{u}\right)$. But there exists a constant $c$ such that

$$
\|u\|_{(M), \dot{a} u} \leq\|u\|_{(M), \Omega} \leq\|u\|_{M, \Omega_{u}}+\|u\|_{M, \Omega \backslash \Omega_{u}} \leq 2\|u\|_{(M), \Omega_{u}}+c
$$

for all $u \in L_{M}(\Omega)$. Consequently the conclusion of the lemma follows from (3.5). Q.E.D.

We conclude this section with the following result where, simultaneously, no global a priori bound is required and no additional structural condition is imposed on the mapping $T$.

Theorem 3.15. Let $\left(Y, Y_{0} ; Z, Z_{0}\right)$ be a complementary system and let $T$ : $Y \rightarrow 2^{Z}$ be a pseudomonotone mapping with respect to a dense subspace $V$ of $Y_{0^{0}}$. Suppose that the graph of $T$ is closed in the following sense: if $\left(y_{i}, z_{i}\right)$ $\epsilon_{\mathrm{gr}} T, y_{i} \rightarrow y \in Y$ for $\sigma\left(Y, Z_{0}\right), y_{i}$ bounded, $z_{i} \rightarrow z \in Z_{0}$ in norm, then $(y, z)$ $\epsilon_{\mathrm{gr}} T$. Suppose that there exists $k>0$ such that, for some $\bar{y} \in V$,

$$
\inf \left\{\langle y-\bar{y}, z\rangle\|y\|^{-1} ; z \in T y\right\} \geq-k
$$

for $y \in D(T)$ with $\|y\|$ sufficiently large, and that $T^{-1}$ is strongly quasibounded on $Z$ with respect to any $z \in Z_{0}$. Suppose also that $Y$ admits an equivalent admissible norm \|\|$_{Y} \cdot$ Then $R(T) \supset Z_{0}$.

Proof. Translating $T$ by a fixed element of $V$, we can assume that $\bar{y}=0$. Let $J$ be the duality mapping corresponding to \|\|$_{Y}$. Define $T_{\epsilon}=T+\epsilon J, \epsilon>0$. By Proposition 2.2, condition (3.6) and Theorem 3.1, $R\left(T_{\epsilon}\right) \supset Z_{0}$ for each $\epsilon>0$. Thus, given $z \in Z_{0}$, we can write $z=u_{\epsilon}+\epsilon v_{\epsilon}$ with $u_{\epsilon} \epsilon T y_{\epsilon}$ and $v_{\epsilon} \epsilon J y_{\epsilon}$. We will show that $y_{\epsilon}$ remains bounded in $Y$ as $\epsilon \downarrow 0$, so that the conclusion follows 
as in the proof of Theorem 3.10. If $\left\|y_{\epsilon}\right\|_{Y}$ is large enough, then, by (3.6),

$$
-k\left\|y_{\epsilon}\right\|_{Y} \leq\left\langle y_{\epsilon}, u_{\epsilon}\right\rangle=\left\langle y_{\epsilon}, z\right\rangle-\epsilon\left\|y_{\epsilon}\right\|_{Y}^{2},
$$

which implies that $\epsilon y_{\epsilon}$ remains bounded in $Y$. Consequently $u_{\epsilon}=z-\epsilon v_{\epsilon}$ remains bounded in $Z$. Since

$$
\left\langle y_{\epsilon}, u_{\epsilon}-z\right\rangle=-\left\langle y_{\epsilon} \in v_{\epsilon}\right\rangle \leq 0
$$

and $T^{-1}$ is strongly quasibounded with respect to $z$, we conclude that $y_{\epsilon}$ remains bounded in $Y$. Q.E.D.

Theorem 3.15 is a variant of both Corollary 3.7 and Theorem 3.10. It will be seen in $\$ 4$ that the mapping $S$ of Example 3.13 satisfies the assumptions of Theorem 3.15. In the sequential version of Theorem 3.15, one requires that $Y_{0}$ and $Z_{0}$ be separable, that $T$ be sequentially pseudomonotone with respect to any dense subspace $V$ of a dense subspace $V^{\prime}$ of $Y_{0}$, that $\bar{y} \in V^{\prime}$ and that the graph of $T$ be sequentially closed.

It may be of interest to compare in a familiar situation the various asymptotic and structural conditions considered in this section. Let $X$ be a reflexive Banach space and let $T: X \rightarrow X^{*}$ be a single-valued mapping which is pseudomonotone with respect to $X$. Then $T$ is onto if one of the following conditions is satisfied:

(1) $T$ is coercive on $X$ with respect to some $\bar{x} \in X$ (Theorem 3.1),

(2) $T^{-1}: X^{*} \rightarrow 2^{X}$ is bounded on $X^{*}$; moreover for some $\bar{x} \in X$ and $k \in \mathbf{R}^{+}$, $\langle x-\bar{x}, T x\rangle \geq-k\|x\|$ for $\|x\|$ sufficiently large (Corollary 3.7),

(3) for some $\bar{x} \in X,\langle x-\bar{x}, T x\rangle\|x\|^{-1}+\|T x\| \rightarrow+\infty$ when $\|x\| \rightarrow \infty$; moreover, for some $b: \mathbf{R}^{+} \rightarrow \mathbf{R}^{+}$continuous, $\langle x-\bar{x}, T x\rangle \geq-b(\|x\|)$ for $\|x\|$ sufficiently large (Theorem 3.5),

(4) $T^{-1}$ is locally bounded on $X^{*}$; moreover $T$ is odd outside some ball of $X$ (Corollary 3.9),

(5) $T^{-1}$ is locally bounded on $X^{*}$; moreover $T$ is monotone (Theorem 3.10),

(6) $T^{-1}$ is strongly quasibounded on $X^{*}$ with respect to any $x^{*} \in X^{*}$; moreover for some $\bar{x} \in X$ and $k \in \mathbf{R}^{+},\langle x-\bar{x}, T x\rangle \geq-k\|x\|$ for $\|x\|$ sufficiently large (Theorem 3.15).

Remark 3.16. In the sequential versions of the theorems of this section, if the mappings are only sequentially of type $(M)$ or sequentially pseudomonotone with respect to one $V$, then the same conclusions hold, with essentially the same proofs, provided the mappings are assumed to be strongly quasibounded.

Remark 3.17. All the applications in $\$ \$ 4$ and 5 could be treated by considering only the case $V=Y_{0}$ (cf. Remarks 4.7, 5.2 and 3.16).

4. Applications I. Under suitable assumptions on the coefficients, systems of the form 


$$
A(u) \equiv \sum_{|\alpha| \leq_{m}}(-1)^{|\alpha|} D^{\alpha} A_{\alpha}\left(x, u, \ldots, \nabla^{m} u\right)
$$

with rapidly (or slowly) increasing coefficients define pseudomonotone mappings, so that the results of $\$ 3$ can be applied. In this section we consider the case where the $A_{a}$ 's satisfy a monotonicity condition with respect to all the derivatives of $u$. For simplicity we discuss a single equation instead of a system. Our results can also easily be extended to "anisotropic" situations where the rate of growth of $A_{a}$ depends on $a$, as considered in [15] (see Example 4.12).

The following notations will be used. If $\xi=\left\{\xi_{a} ;|a| \leq m\right\} \in \mathbf{R}^{s_{m}}$ is an m-jet, with $a=\left(a_{1}, \cdots, a_{n}\right)$ a multi-index of integers and $|a|=a_{1}+\cdots+a_{n}$, then $\zeta=\left\{\xi_{a} ;|a|=m\right\} \in \mathbf{R}^{s_{m}^{\prime}}$ denotes its top order part and $\eta=\left\{\xi_{a} ;|a|<m\right\} \in \mathbf{R}^{s_{m-1}}$ its lower order part. For $u$ a derivable function, $\xi(u)$ denotes $\left\{D^{a} u ;|a| \leq m\right\}$. The nonnegative reciprocal function of a $N$-function $M$ is denoted by $M^{-1}$, $N \prec M$ means that there exists $k$ such that $N(t) \leq M(k t)$ for large values of $t$, and $N \ll M$ means that for each $\epsilon>0, N(t) / M(\epsilon t) \rightarrow 0$ as $t \rightarrow \infty$. One has $N \prec M$ if and only if $\bar{M} \prec \bar{N}$, and $N \ll M$ if and only if $\bar{M} \ll \bar{N}$. $P\left(E_{M}, r\right)$ denotes those $u$ in $L_{M}(\Omega)$ whose distance to $E_{M}(\Omega)$ (with respect to the Orlicz norm) is strictly less than $r$ and $B_{r}\left(0, L_{M}\right)$ the ball in $L_{M}(\Omega)$ (with respect to the Orlicz norm) of radius $r$ and center 0 . The inclusions $\mathscr{P}\left(E_{M}, 1\right) \subset \mathscr{L}_{M}(\Omega)$ and $L_{M}(\Omega) \subset E_{N}(\Omega)$ for $N \ll M$ hold (cf. [24]).

Let $\Omega$ be an open subset of $R^{n}$. The basic conditions imposed on the coefficients $A_{a}$ of (4.1) are

(4.2) Carathéodory condition. Each $A_{a}(x, \xi)$ is a real-valued function defined on $\Omega \times \mathbf{R}^{s_{m}}$ which is measurable in $x$ for fixed $\xi$ and continuous in $\xi$ for fixed $x$.

(4.3) Growth condition. There exists an $N$-function $M, a(x) \in E_{\bar{M}}(\Omega)$ and $b$, $c \in \mathbf{R}^{+}$such that, for all $|\alpha| \leq m, x \in \Omega$ and $\xi \in \mathbf{R}^{s_{m}}$,

$$
\left|A_{a}(x, \xi)\right| \leq a(x)+b \sum_{|\beta| \leq m} \bar{M}^{-1} M\left(c \xi_{\beta}\right) .
$$

(4.4) Monotonicity condition. For all $x \in \Omega$ and $\xi, \xi^{\prime} \in \mathbf{R}^{s_{m}}$,

$$
\sum_{|a| \leq m}\left(A_{a}(x, \xi)-A_{a}\left(x, \xi^{\prime}\right)\right)\left(\xi_{a}-\xi_{a}^{\prime}\right) \geq 0 .
$$

Let $Y$ be a $\sigma\left(\Pi L_{M}, \Pi E_{\bar{M}}\right)$ closed subspace of $W^{m} L_{M}(\Omega)$ such that

$$
Y=\sigma\left(\prod L_{M}, \Pi L_{\bar{M}}\right) \mathrm{cl} Y_{0}
$$

where $Y_{0}=Y \cap W^{m} E_{M}(\Omega)$; here $M$ is the $N$-function involved in (4.3). Let $f \epsilon$ $Y_{0}^{*}$. The Variational Boundary Value Problem for $A(u)=f$ witb respect to $Y$ asks 
for an element $u \in Y$ such that $A_{a}(\xi(u)) \in L_{\bar{M}}(\Omega)$ for all $|a| \leq m$ and $a(u, v)=$ $f(v)$ for all $v \in Y_{0}$, where

$$
a(u, v) \equiv \int_{\Omega} \sum_{|a| \leq_{m}} A_{\alpha}(\xi(u)) D^{a} v d x
$$

is the Dirichlet form associated with $A$. The analytical fact which allows the application of the results of $\S_{3}$ to this problem is contained in

Theorem 4.1. Let $A$ be an operator of the form (4.1) satisfying (4.2), (4.3) and (4.4). Let $Y$ be a $\sigma\left(\Pi L_{M}, \Pi E_{\bar{M}}\right)$ closed subspace of $W^{m} L_{M}(\Omega)$ satisfying (4.5) and let $\left(Y, Y_{0} ; Z, Z_{0}\right)$ be the complementary system generated by $Y$ in (П $L_{M}$, П $E_{M} ; \Pi L_{\bar{M}}$, П $E_{\bar{M}}$ ). Let $T$ be the mapping from

$$
D(T)=\left\{u \in Y ; A_{a}(\xi(u)) \in L_{\bar{M}}(\Omega) \text { for all }|a| \leq m\right\}
$$

into $Z$ defined by $\langle v, T u\rangle=a(u, v)$ for all $v \in Y_{0}$. Then $T$ is pseudomonotone with respect to any dense subspace $V$ of $Y_{0}$.

Remark 4.2. By means of an argument similar to that used at the beginning of the proof of Theorem 4.1 below, one can see that $D(T)=\left\{u \in Y ; \Sigma_{|a| \leq m} A_{a}(\xi(u)) D^{a} u\right.$ $\left.\epsilon L^{1}(\Omega)\right\}$. Note also that (4.5) implies that $\langle v, T u\rangle=a(u, v)$ for all $u \in D(T)$ and $v \in Y$.

The following lemmas will be needed in the proof of Theorem 4.1. In relation with Lemma 4.3, we remark that the continuity result of $[24$, p. 170] cannot be applied here to derive the finite continuity of $T$. Lemma.4.5 is concerned with maximal monotonicity; it generalizes a result of Donaldson [12, p. 519].

Lemma 4.3. Suppose that (4.2) and (4.3) bold (with $a(x) \in L_{\bar{M}}(\Omega)$ ). Then the mapping $w=\left(w_{\beta}\right)_{|\beta| \leq m} \mapsto\left(A_{\alpha}(w)\right)_{|\alpha|_{\leq m}}$ sends $\Pi E_{M}$ into $\Pi L_{\bar{M}}$ and is finitely continuous from $\Pi E_{M}$ to the $\sigma\left(\Pi L_{\bar{M}}, \Pi E_{M}\right)$ topology of $\Pi L_{\bar{M}}$.

Proof. It is immediate that $A_{a}(w) \in L_{\bar{M}}(\Omega)$ if $w \in \Pi E_{M}$. We will show that the mapping is continuous from each simplex in $\Pi E_{M}$ to the $\sigma\left(\Pi L_{\bar{M}}, \Pi E_{M}\right)$ topology of $\Pi L_{\bar{M}}$. Let $S=\operatorname{conv}\left\{w^{1}, \ldots, w^{r}\right\}$ be a simplex in $\Pi E_{M}$ and write $w=\Sigma_{i=1}^{r} \lambda_{i} w^{i} \in S$ with $\lambda_{i} \geq 0$ and $\Sigma_{i=1}^{r} \lambda_{i}=1$. We have

$$
\bar{M}^{-1} M\left(c w_{\beta}\right)=\bar{M}^{-1} M\left(\sum_{i=1}^{r} \lambda_{i} c w_{\beta}^{i}\right) \leq \bar{M}^{-1}\left(\sum_{i=1}^{r} \lambda_{i} M\left(c w_{\beta}^{i}\right)\right),
$$

which implies that each $A_{a}(w)$ remains bounded in $L_{\bar{M}}(\Omega)$ when $w$ runs over $S$. It is then easy to complete the proof by means of the following lemma. Q.E.D. 
Lemma 4.4 (cf. [24, p. 132]). If a sequence $g_{n} \in L_{N}(\Omega)$ converges a.e. to $g$ and if $g_{n}$ remains bounded in $L_{N}(\Omega)$, then $g \in L_{N}(\Omega)$ and $g_{n} \rightarrow g$ for $\sigma\left(L_{N}, E_{\bar{N}}\right)$.

Lemma 4.5. Suppose that (4.2), (4.3) and (4.4) bold (with $a(x) \in L_{\bar{M}}(\Omega)$ ). Let $g \in \Pi L_{M}$ and $b \in \Pi L_{\bar{M}}$ satisfy

$$
\int_{\Omega} \sum_{|\alpha| \leq m}\left(A_{\alpha}(w)-b_{\alpha}\right)\left(w_{\alpha}-g_{\alpha}\right) d x \geq 0
$$

for all $w \in \Pi L^{\infty}$ with compact. support in $\bar{\Omega}$. Then $A_{a}(g)=b_{a}$ for all $|\alpha| \leq m$.

Proof. Let $\Omega_{k}=\left\{x \in \Omega ;|x| \leq k\right.$ and $\left|g_{a}(x)\right| \leq k$ for all $\left.|a| \leq m\right\}$. Clearly, $\Omega_{k} \subset \Omega_{k+1}$, and for each ball $B$, meas $\left(B \cap\left(\Omega \backslash \Omega_{k}\right)\right) \rightarrow 0$ as $k \rightarrow \infty$. Denote by $\chi_{k}$ the characteristic function of $\Omega_{k}$. Fix $k$ and replace $w$ in (4.6) by $w \chi_{k}-$ $g \chi_{k}+g X_{l} \equiv w^{k}-g^{k}+g^{l}$, where $l \geq k$ :

$$
\begin{aligned}
0 \leq & \int_{\Omega} \sum\left(A_{\alpha}\left(w^{k}-g^{k}+g^{l}\right)-b_{\alpha}\right)\left(w_{\alpha}^{k}-g_{\alpha}^{k}+g_{\alpha}^{l}-g_{\alpha}\right) d x \\
= & \int_{\Omega} \sum\left(A_{\alpha}\left(w^{k}-g^{k}+g^{l}\right)-A_{\alpha}(0)\right)\left(g_{\alpha}^{l}-g_{\alpha}\right) d x \\
& -\int_{\Omega} \sum\left(b_{\alpha}-A_{\alpha}(0)\right)\left(g_{\alpha}^{l}-g_{\alpha}\right) d x \\
& +\int_{\Omega} \sum\left(A_{\alpha}\left(w^{k}-g^{k}+g^{l}\right)-b_{\alpha}\right)\left(w_{\alpha}^{k}-g_{\alpha}^{k}\right) d x .
\end{aligned}
$$

The first integral of the right-hand side is zero because $A_{a}\left(w^{k}-g^{k}+g^{l}\right)$ $A_{\alpha}(0)=0$ outside $\Omega_{l}$ and $g_{a}^{l}-g_{a}=0$ in $\Omega_{l}$, the second integral goes to zero as $l \rightarrow+\infty$, and the last integral is equal to

$$
\int_{Q} \sum\left(A_{a}\left(w^{k}-g^{k}+g^{k}\right)-b_{a}\right)\left(w_{a}^{k}-g_{a}^{k}\right) d x
$$

because $w_{a}^{k}-g_{a}^{k}=0$ outside $\Omega_{k}$. Hence, letting $l \rightarrow \infty$, we obtain

$$
\int_{\Omega} \sum\left(A_{a}\left(w^{k}\right)-b_{\alpha}\right)\left(w_{a}^{k}-g_{a}^{k}\right) d x \geq 0
$$

for all $w \in \Pi L^{\infty}$ with compact support in $\bar{\Omega}$. Applying Minty's classical argument [30] to the mapping $w \in \Pi L^{\infty}\left(\Omega_{k}\right) \mapsto\left(A_{\alpha}(w)\right) \in \Pi L_{\bar{M}}\left(\Omega_{k}\right)$ which is everywhere defined, monotone and finitely continuous by Lemma 4.3 , we deduce from (4.7) that $A_{a}(g)=b_{a}$ a.e. in $\Omega_{k}$ for all $|a| \leq m$. Since $k$ was arbitrary, $A_{\alpha}(g)=b_{\alpha}$ a.e. in $\Omega$ for all $|\alpha| \leq m$. Q.E.D.

Remark 4.6. Suppose that $E_{\bar{M}}(\Omega)=L_{\bar{M}}(\Omega)$ and write $X=\Pi E_{M}, X^{*}=\Pi L_{\bar{M}}$, $X^{* *}=\Pi L_{M}$. Then, using the method of Lemma 4.5, one can show that the map- 
ping $S: X \rightarrow X^{*}: w \mapsto\left(A_{a}(w)\right)$ satisfies the assumption of Example 3.2: $S_{1}$ is maximal monotone (and is given by $S_{1}: D\left(S_{1}\right) \subset X^{* *} \rightarrow X^{*}: w \mapsto\left(A_{a}(w)\right)$ with $D\left(S_{1}\right)=\left\{w \in X^{* *} ; A_{a}(w) \in L_{\bar{M}}(\Omega)\right.$ for all $\left.\left.|\alpha| \leq m\right\}\right)$. It is not known whether the analogous result is true for the mapping $\left.T\right|_{Y_{0}}: Y_{0} \rightarrow Y_{0}^{*}$ of Theorem 4.1 (the answer is yes when $\left.T\right|_{Y_{0}}$ is the gradient of a convex functional on $Y_{0}$, cf. [16]). It is not even known whether the mapping $T: D(T) \subset Y_{0}^{* *} \rightarrow Y_{0}^{*}$ is always maximal monotone (cf. [17]).

Proof of Theorem 4.1. Let $V$ be a dense subspace of $Y_{0}$. By Lemma 4.3, $V \subset D(T)$ and $T$ is finitely continuous from $V$ to the $\sigma(Z, V)$ topology of $Z$. Let $u_{i}$ be a net with $u_{i} \in V, u_{i}$ bounded, $u_{i} \rightarrow u \in Y$ for $\sigma\left(Y, Z_{0}\right), T u_{i} \rightarrow f \in Z$ for $\sigma\left(Z, V_{0}\right)$, and

$$
\lim \sup \left\langle u_{i}, T u_{i}\right\rangle \leq\langle u, f\rangle .
$$

We must prove that $u \in D(T), T u=f$ and $\left\langle u_{i}, T u_{i}\right\rangle \rightarrow\langle u, f\rangle$. As usual, one can pass to a subnet if necessary.

First we show that for each $|a| \leq m, A_{a}\left(\xi\left(u_{i}\right)\right)$ remains bounded in $L_{\bar{M}}(\Omega)$. Let $w=\left(w_{\beta}\right) \in \Pi E_{M}$. We have

$$
\int_{\Omega} \sum\left(A_{\alpha}\left(\xi\left(u_{i}\right)\right)-A_{\alpha}(w)\right)\left(D^{\alpha} u_{i}-w_{\alpha}\right) d x \geq 0
$$

i.e.,

$$
\begin{aligned}
& \int_{\Omega} \sum A_{\alpha}\left(\xi\left(u_{i}\right)\right) w_{\alpha} d x \\
& \quad \leq \int_{\Omega} \sum A_{\alpha}\left(\xi\left(u_{i}\right)\right) D^{\alpha} u_{i} d x+\int_{Q} \sum A_{\alpha}(w) w_{\alpha} d x-\int_{\Omega} \sum A_{\alpha}(w) D^{\alpha} u_{i} d x_{0}
\end{aligned}
$$

The first integral of the right-hand side remains bounded from above by (4.8) (if $i \geq$ some $i_{0}$ ), the second integral is independent of $i$ and the last integral remains bounded. Hence, passing to a trivial subnet, each $A_{a}\left(\xi\left(u_{i}\right)\right)$ remains bounded in $L_{\bar{M}}(\Omega)$ for $\sigma\left(L_{\bar{M}}, E_{M}\right)$, i.e., remains bounded in $L_{\bar{M}}(\Omega)$.

Consequently, we can assume that each $A_{a}\left(\xi\left(u_{i}\right)\right) \rightarrow b_{a} \in L_{\bar{M}}(\Omega)$ for $\sigma\left(L_{\bar{M}}, E_{M}\right)$. It follows that the linear form $f \in Z=Y_{0}^{*}$ can be identified to $\left(b_{a}\right) \in$ ח $L_{\bar{M}}$. More precisely, the action of $f$ over $V$ is clearly given by

$$
\langle v, f\rangle=\int_{\Omega} \sum b_{a} D^{a} v d x, \quad v \in V
$$

since $V$ is dense in $Y_{0}$ and $Y_{0}$ satisfies (4.5), this formula also describes the action of $f$ over $Y$, i.e., holds for $v \in Y$. Now going to the limit in (4.9) and using the facts that $\sigma\left(\Pi L_{M}, \Pi E_{\bar{M}}\right)$ induces $\sigma\left(Y, Z_{0}\right)$ on $Y$ and $a(x) \in E_{\bar{M}}(\Omega)$, we obtain 


$$
\int_{Q} \sum\left(b_{a}-A_{a}(w)\right)\left(D^{a} u-w_{a}\right) d x \geq 0
$$

for all $w=\left(w_{\beta}\right) \in \Pi L^{\infty}$ with compact support in $\bar{\Omega}$. It then follows from Lemma 4.5 that $A_{a}(\xi(u))=b_{a}$ for all $|a| \leq m$, i.e. that $u \in D(T)$ and $T u=f$.

To prove that $\left\langle u_{i}, T u_{i}\right\rangle \rightarrow\langle u, f\rangle$, we first deduce from (4.9) that

$$
\begin{aligned}
L & \equiv \lim \inf \int_{\Omega} \sum A_{a}\left(\xi\left(u_{i}\right)\right) D^{\alpha} u_{i} d x \\
& \geq \int_{\Omega} \sum A_{a}(w)\left(D^{a} u-w_{\alpha}\right) d x+\int_{Q} \sum A_{a}(\xi(u)) w_{a} d x
\end{aligned}
$$

for all $w=\left(w_{\beta}\right) \in \Pi L^{\infty}$ with compact support in $\bar{\Omega}$. Let $\Omega_{k}=\{x \in \Omega ;|x| \leq k$ and $\left|D^{\alpha} u(x)\right| \leq k$ for all $\left.|a| \leq m\right\}$ and denote as above by $\chi_{k}$ the characteristic function of $\Omega_{k}$. Then

$$
\begin{aligned}
L \geq & \int_{\Omega} \sum\left(A_{\alpha}\left(\chi_{k} \xi(u)\right)-A_{\alpha}(0)\right)\left(D^{\alpha} u-\chi_{k} D^{\alpha} u\right) d x \\
& +\int_{\Omega} \sum A_{a}(0)\left(D^{\alpha} u-\chi_{k} D^{\alpha} u\right) d x+\int_{\Omega} \sum A_{\alpha}(\xi(u)) \chi_{k} D^{a} u d x,
\end{aligned}
$$

where the first integral is zero. Letting $k \rightarrow+\infty$, we get

$$
L \geq \int_{\Omega} \sum A_{\alpha}(\xi(u)) D^{\alpha} u d x .
$$

This inequality and (4.8) imply $\left\langle u_{i}, T u_{i}\right\rangle \rightarrow\langle u, f\rangle$. Q.E.D.

Remark 4.7. The arguments in the above proof show that the graph of $T$ in Theorem 4.1 is closed with respect to the convergence involved in Theorems 3.10 and 3.15. They also show that $T$ is strongly quasibounded on $Y$ with respect to any $y \in Y_{0^{\circ}}$. Similar remarks apply to Theorem 4.15 .

The combination of Theorem 4.1 with the results of $\S_{3}$ leads to several existence theorems for (4.1). For instance:

Theorem 4.8. Let $A$ be an operator of the form (4.1) satisfying (4.2), (4.3) and (4.4). Let $Y$ be a $\sigma\left(\Pi L_{M}\right.$, $\left.\Pi E_{\bar{M}}\right)$ closed subspace of $W^{m} L_{M}(\Omega)$ satisfying (4.5). Suppose that a $(u, u)\|u\|_{m,(M)}^{-1} \rightarrow+\infty$ as $\|u\|_{m_{0}(M)} \rightarrow+\infty$ in $Y_{0^{*}}$.Tben, for eacb $\sigma\left(\Pi E_{M}, \Pi E_{\bar{M}}\right)$ continuous linear form $f$ on $Y_{0}$, the V.B.V.P. for $A(u)$ $=f$ with respect to $Y$ has at least one solution.

Theorem 4.9. Let $A$ be an operator of the form (4.1) satisfying (4.2), (4.3) and (4.4). Let $Y$ be a $\sigma\left(\Pi L_{M}\right.$, $\left.\Pi E_{\bar{M}}\right)$ closed subspace of $W^{m} L_{M}(\Omega)$ satisfying (4.5). Suppose that for eacb $\sigma\left(\Pi E_{M}, \Pi E_{\bar{M}}\right)$ continuous linear form $f$ on $Y_{0}$ there exist a constant $K$ and a neighbourbood $r$ of $f$ in $Y_{0}^{*}$ sucb that for any $g \in \Re$ and any solution $u$ of the V.B.V.P. for $A(u)=g$ with respect to $Y$, 
$\|u\|_{m_{0}(M)} \leq K$. Then, for each $\sigma\left(\Pi E_{M}, \Pi E_{\bar{M}}\right)$ continuous linear form $f$ on $Y_{0}$, the V.B.V.P. for $A(u)=f$ with respect to $Y$ has at least one solution.

Remark 4.10. If condition (4.4) is strenghthened to

$$
\sum_{|a| \leq m}\left(A_{a}(x, \xi)-A_{a}\left(x, \xi^{\prime}\right)\right)\left(\xi_{a}-\xi_{a}^{\prime}\right)>0
$$

for all $x \in \Omega$ and $\xi, \xi^{\prime} \in \mathbf{R}^{s_{m}}$ with $\xi \neq \xi^{\prime}$, then the mapping $T$ is strictly monotone, and the solutions in Theorems 4.8 and 4.9 are unique.

Concrete analytical conditions on the coefficients $A_{a}$ implying coercivity or a local a priori bound can be deduced from Lemma 3.14 or from the treatment of the following example respectively.

Example 4.11. Consider the Dirichlet problem for the operator

$$
A(u) \equiv \sum_{|a| \leq m}(-1)^{|a|} D^{a}\left(p\left(D^{a} u\right)\right)
$$

on an open subset $\Omega$ of $\mathbf{R}^{n}$ with the segment property. Here $p: \mathbf{R} \rightarrow \mathbf{R}$ is a nondecreasing odd continuous function with $p(+\infty)=+\infty$. Write $M(t)=\int_{0}^{t} p(s) d s$. Then the assumptions (4.2), (4.3), (4.4) of Theorem 4.9 are easily verified, and Theorem 1.3 implies that $Y=W_{0}^{m} L_{M}(\Omega)$ satisfies (4.5). The dual $Z$ of $Y_{0}=$ $W_{0}^{m} E_{M}(\Omega)$ is

$$
W^{-m} L_{\bar{M}}(\Omega)=\left\{f \in \mathscr{D}^{\prime}(\Omega) ; f=\sum_{|\alpha| \leq_{m}}(-1)^{|\alpha|} D^{\alpha} f_{a} \text { with } f_{a} \in L_{\bar{M}}(\Omega)\right\},
$$

and the subspace $Z_{0}$ of $Y_{0}^{*}$ consisting of those linear forms on $Y_{0}$ which are $\sigma\left(\Pi E_{M}, \Pi E_{\bar{M}}\right)$ continuous is

$$
W^{-m} E_{\bar{M}}(\Omega)=\left\{f \in \mathscr{I}^{\prime}(\Omega) ; f=\sum_{|\alpha| \leq m}(-1)^{|\alpha|} D^{\alpha} f_{a} \text { with } f_{\alpha} \in E_{\bar{M}}(\Omega)\right\} .
$$

To verify the local a priori bound, let $f=\left(f_{a}\right) \in Z_{0}$. Choose $c$ such that $\int_{\Omega} \bar{M}\left(2 f_{a}\right) d x \leq c$ for all $|a| \leq m$, and define

$$
r=\left\{g=\left(g_{\alpha}\right) \in Z ; \int_{\mathbb{Q}} \bar{M}\left(2 g_{a}\right) \leq c+1 \text { for all }|\alpha| \leq m\right\} \text {. }
$$

$\gamma$ is a neighbourhood of $f$ in $Z$ since the functional $a(x) \rightarrow \int_{\Omega} \bar{M}(a(x)) d x$ is continuous on $\mathscr{P}\left(E_{\bar{M}}, 1\right)$ (because it is convex on $L_{\bar{M}}(\Omega)$, finite on $\mathcal{L}_{\bar{M}}(\Omega) \supset$ $\mathcal{P}\left(E_{\bar{M}}, 1\right)$ and bounded from above on $B_{1}\left(0, L_{\bar{M}}\right)$, cf. $[24$, p. 74$\left.]\right)$. We claim that the possible solutions of the V.B.V.P. for $A(u)=g$ with respect to $Y$ remain bounded in $Y$ as $g \in \Re$. Indeed, if $u$ is such a solution, then

$$
\int_{\Omega} \sum p\left(D^{a} u\right) D^{a} v d x=\int_{\Omega} \sum g_{a} D^{a} v d x
$$


for all $v \in Y_{0}$, and thus for all $v \in Y$ since (4.5) holds. In particular,

$$
\int_{\mathbf{Q}} \sum p\left(D^{a} u\right) D^{a} u d x=\int_{a} \sum g_{a} D^{a} u d x .
$$

The left-hand side is greater than $\int_{\Omega} \Sigma M\left(D^{\alpha} u\right) d x$, and the right-hand side is less than

$$
\int_{\Omega} \sum \bar{M}\left(2 g_{\alpha}\right) d x+\int_{\Omega} \sum M\left(\frac{1}{2} D^{\alpha} u\right) d x \leq c^{\prime}+\frac{1}{2} \int_{\Omega} \sum M\left(D^{a} u\right) d x .
$$

Consequently,

$$
\int_{\mathbb{\Omega}} \sum_{|a| \leq m} M\left(D^{\alpha} u\right) d x \leq 2 c^{\prime}
$$

which implies that $u$ remains bounded in $Y$. Theorem 4.9 can thus be applied: for each $f \in W^{-m} E_{\bar{M}}(\Omega)$, the V.B.V.P. for $A(u)=f$ with respect to $W_{0}^{m} L_{M}(\Omega)$ has at least one solution.

Example 4.12. We indicate briefly a simple anisotropic situation to which the method of Theorem 4.9 can be applied. Consider the Dirichlet problem for the operator

$$
A(u) \equiv \sum_{|\alpha| \leq_{m}}(-1)^{|\alpha|} D^{\alpha}\left(p_{\alpha}\left(D^{\alpha} u\right)\right)
$$

on a bounded open subset $\Omega$ of $\mathbf{R}^{n}$ with the segment property. For each $a, p_{a}$ : $\mathbf{R} \rightarrow \mathbf{R}$ is a nondecreasing odd continuous function with $p_{a}(+\infty)=+\infty$. Write $M_{a}(t)=\int_{0}^{t} p_{a}(s) d s$ and consider the complementary system $\left(\Pi L_{M_{a}}, \Pi E_{M_{a}}\right.$; $\left.\Pi L_{\bar{M}_{a}}, \Pi E_{\bar{M}_{a}}\right)$ and the space $Y=\sigma\left(\Pi L_{M_{\alpha}}\right.$, $\left.\Pi E_{\bar{M}_{\alpha}}\right) \mathrm{cl} \mathscr{D}(\Omega)$. If $M_{a}>M_{\beta}$ for $\alpha \leq \beta$ (i.e. $\alpha_{i} \leq \beta_{i}$ for each $\left.i=1, \ldots, n\right)$, then $Y_{0}=Y \cap \Pi E_{M_{a}}$ is $\sigma\left(\Pi L_{M_{a}}, \Pi L_{\bar{M}_{a}}\right.$ ) dense in $Y$. The proof is similar to that of Theorem 1.3; the assumptions $\Omega$ bounded and $M_{a}>M_{\beta}$ for $a \leq \beta$ (which can be slightly weakened using Proposition 4.13 below) are needed in order to apply Leibnitz's formula. The arguments of Theorem 4.1 and Example 4.11 carry over with little change, and we obtain: for each $f \in \mathscr{D}^{\prime}(\Omega)$ which can be written as

$$
f=\sum_{|\alpha| \leq m}(-1)^{|\alpha|} D^{\alpha} f_{a}
$$

with $f_{a} \in E_{\bar{M}_{a}}(\Omega)$, there exists $u \in Y$ such that $p_{a}\left(D^{a} u\right) \in L_{\bar{M}_{a}}(\Omega)$ for all $|a| \leq m$ and $a(u, v)=f(v)$ for all $v \in Y_{0}$. In the case where $p_{a}$ has polynomial growth for $|a|=m$, the result of this example is related, although different, to the existence theorems of Browder [9] and Hess [22], [23] about equations with strongly nonlinear lower order terms. 
We now turn to the case where $\Omega$ is bounded with, say, a locally Lipschitzian boundary so that the generalized version of the Sobolev imbedding theorem [13] is valid on $\Omega$. Then the growth condition (4.3) can be weakened and a lower order perturbation can be introduced.

First we recall the result of [13]. Let $C_{0}$ be an $N$-function and suppose first $n \geq 2$. Changing the values of $C_{0}$ on a bounded subset of $R$, one can assume $\int_{0}^{1} C_{0}^{-1}(t) / t^{1+1 / n} d t<+\infty$. If $\int_{1}^{\infty} C_{0}^{-1}(t) / t^{1+1 / n} d t=+\infty$, define a new $N$-function $C_{1}$ by $C_{1}^{-1}(s)=\int_{0}^{s} C_{0}^{-1}(t) / t^{1+1}{ }_{n} d t$. Repeating this process, one obtains a finite sequence of $N$-functions $C_{0}, C_{1}, \ldots, C_{q}$, where $q=q\left(C_{0}\right) \leq n$ is such that $\int_{1}^{\infty} C_{q-1}^{-1}(t) / t^{1+1 / n} d t=+\infty$ but $\int_{1}^{\infty} C_{q}^{-1}(t) / t^{1+1 / n} d t<+\infty$. If $n=1$, write $q\left(C_{0}\right)=0$.

Proposition 4.13 (cf. [13]). Let $\Omega$ be a bounded open subset of $\mathbf{R}^{\boldsymbol{n}}$ with locally Lipscbitzian boundary. Let $M$ be an $N$-function and let $u \in W^{m} L_{M}(\Omega)$. For $m-q(M) \leq|a| \leq m$, write $M_{a}=C_{m-|a|}$ starting witb $C_{0}=M$. Then (a) for $m-q(M) \leq|a| \leq m, D^{a} u \in L_{M a}(\Omega)$ with continuous injection, and $D^{a}{ }_{u} \in E_{N}(\Omega)$ with compact injection if $N \ll M_{a}$; (b) for $|a|<m-q(M), D^{a}{ }_{u} \in C(\bar{\Omega})$ with compact injection. Moreover, if $u \in W^{m} E_{M}(\Omega)$, then in (a), $D^{a} u \in E_{M a}(\Omega)$.

The following lemma completes Proposition 4.13. It implies that the injection of $W^{m} L_{M}(\Omega)$ into $W^{m-1} L_{M}(\Omega)$. is compact.

Lemma 4.14. Let $0<\epsilon<1$ and let $C_{0}$ be a $N$-function sucb that $\int_{0}^{1} C_{0}^{-1}(t) / t^{1+\epsilon} d t<+\infty$ and $\int_{1}^{\infty} C_{0}^{-1}(t) / t^{1+\epsilon} d t=+\infty$. Then tbe $N$-function $C_{1}$ defined by $C_{1}^{-1}(s)=\int_{0}^{s} C_{0}^{-1}(t) / t^{1+\epsilon} d t$ satisfies $C_{0} \ll C_{1}$.

Proof. It is easy to verify that, in general, $C_{0} \ll C_{1}$ if and only if $C_{1}^{-1}(s) / C_{0}^{-1}(s) \rightarrow 0$ as $s \rightarrow+\infty$. We have here

$$
\begin{aligned}
& \frac{1}{C_{0}^{-1}(s)} \int_{0}^{s} C_{0}^{-1}(t) / t^{1+\epsilon} d t \\
& \quad=\frac{1}{C_{0}^{-1}(s)} \int_{0}^{1} C_{0}^{-1}(t) / t^{1+\epsilon} d t+\frac{1}{C_{0}^{-1}(s)} \int_{1}^{s} C_{0}^{-1}(t) / t^{1+\epsilon} d t .
\end{aligned}
$$

The first term on the right-hand side $\rightarrow 0$ as $s \rightarrow \infty$. Writing $t=C_{0}(r)$, the second term becomes

$$
\begin{aligned}
\frac{1}{C_{0}^{-1}(s)} \int_{C_{0}^{-1}(1)}^{C_{0}^{-1}(s)} & r C_{0}^{\prime}(\tau) / C_{0}(\tau)^{1+\epsilon} d r \\
& =\frac{-1}{\epsilon C_{0}^{-1}(s)} \int_{C_{0}^{-1}(1)}^{C_{0}^{-1}(s)} r\left(1 / C_{0}(\tau)^{\epsilon}\right)^{\prime} d r \\
& =\frac{-1}{\epsilon C_{0}^{-1}(s)}\left[\tau / C_{0}^{\left.(\tau)^{\epsilon}\right]} C_{0}^{C_{0}^{-1}(s)}+\frac{1}{\epsilon C_{0}^{-1}(s)} \int_{C_{0}^{-1}(1)}^{C_{0}^{-1}(s)} 1 / C_{0}(\tau)^{\epsilon} d r .\right.
\end{aligned}
$$


The first term of the right-hand side $\rightarrow 0$ as $s \rightarrow \infty$. Since $C_{0}(\tau) \geq \tau$ for $r \geq \tau_{0}$, the second term is less than

$$
\frac{1}{\epsilon C_{0}^{-1}(s)} \int_{C_{0}^{-1}(1)}^{\tau_{0}} 1 / C_{0}(\tau)^{\epsilon} d \tau+\frac{1}{\epsilon C_{0}^{-1}(s)} \int_{\tau_{0}}^{C_{0}^{-1}(s)} 1 / \tau^{\epsilon} d \tau
$$

which clearly $\rightarrow 0$ as $s \rightarrow \infty$. Q.E.D.

With the notations of Proposition 4.13, the new growth condition imposed on the coefficients of (4.1) is

(4.10) There exist a $N$-function $M, N$-functions $N_{a}$ satisfying $M \prec N_{a}$ for $|\alpha| \leq m$ and $N_{a} \prec M_{a}$ for $m-q(M) \leq|\alpha| \leq m, a_{a}(x) \in E_{\bar{N}_{a}}(\Omega)$ for $|\alpha| \leq m$ and $b, c \in \mathbf{R}^{+}$such that, for all $|a| \leq m, x \in \Omega$ and $\xi \in \mathbf{R}^{s_{m}}$,

$$
\left|A_{a}(x, \xi)\right| \leq a_{a}(x)+b \sum_{|\beta| \leq m} \bar{N}_{\alpha}^{-1} N_{\beta}\left(c \xi_{\beta}\right) .
$$

(Note that the $N$-functions $N_{a}$ for $|a|<m-q(M)$ can always be replaced by greater $N$-functions. Combining this with the fact that any given $L^{1}$ function on $\Omega$ belongs to some $E_{N}(\Omega)$ (cf. [24, p. 60]), we see that $(4.10)$ holds as soon as an analogous condition with only $a_{a}(x) \in L^{1}(\Omega)$ for $|a|<m-q(M)$ holds. Similar remarks apply to (5.1) in the next section.)

On the lower order perturbation

$$
\left.B(u) \equiv \sum_{|\gamma| \leq m-1}(-1)^{|\gamma|}\right|_{D^{\gamma}}{ }_{\gamma}\left(x, u, \ldots, \nabla^{m-1} u\right),
$$

we impose the related conditions

(4.12) Each $B_{\gamma}(x, \eta)$ is a real-valued function defined on $\Omega \times R^{s_{m-1}}$ which is measurable on $x$ for fixed $\eta$ and continuous in $\eta$ for fixed $x$.

(4.13) There exist $N$-functions $P_{\gamma}$ satisfying $P_{\gamma} \ll M_{\gamma}$ for $m-q(M) \leq|\gamma|$ $\leq m-1, d_{\gamma}(x) \in E_{\bar{M}_{\gamma}}(\Omega), d(x) \in L^{1}(\Omega), e \in C\left(\mathbf{R}^{s_{m-q}(M)-1}\right)$ and $c \in \mathbf{R}^{+}$such that for all $x \in \Omega$ and $\eta \in \mathbf{R}^{s_{m-1}}$ with component $\eta^{q}$ in $\mathbf{R}^{s_{m-q(M)-1}}$,

if $m-q(M) \leq|\gamma| \leq m-1$,

$$
\left|B_{\gamma}(x, \eta)\right| \leq e\left(\eta^{q}\right)\left[d_{\gamma}(x)+\sum_{m-q(M) \leq|\delta| \leq m-1} \bar{P}_{\gamma}^{-1} P_{\delta}\left(c \eta_{\delta}\right)\right] ;
$$

if $|\gamma|<m-q(M)$,

$$
\left|B_{\gamma}(x, \eta)\right| \leq e\left(\eta^{q}\right)\left[d(x)+\sum_{m-q(M) \leq|\delta| \leq m-1} P_{\delta}\left(c \eta_{\delta}\right)\right] .
$$

Denote $\Pi_{|a|_{\leq m}} L_{N a}(\Omega)$ by $\Pi L_{N_{a}}$. Let $Y$ be a $\sigma\left(\Pi L_{N a}, \Pi E_{\bar{N}_{a}}\right)$ closed subspace of $W^{m} L_{M}(\Omega)$ such that

$$
Y=\sigma\left(\prod L_{N_{a}}, \Pi L_{\bar{N}_{a}}\right) \operatorname{cl} Y_{0}
$$


where $Y_{0}=Y \cap W^{m} E_{M}(\Omega)=Y \cap \Pi E_{N_{a}}$. For instance, by a simple generalization of Theorem 1.3, $Y=W^{m} L_{M}(\Omega)$ or $Y=W_{0}^{m} L_{M}(\Omega)$ satisfy (4.14). Let $f \in Y_{0}^{*}$. The V.B.V.P. for $A(u)+B(u)=f$ with respect to $Y$ asks for an element $u \in Y$ such that $A_{a}(\xi(u)) \in L_{\bar{N}_{\alpha}}(\Omega)$ for all $|\alpha| \leq m$ and $a(u, v)+b(u, v)=$ $f(v)$ for all $v \in Y_{0}$, where $a(u, v)^{\alpha}$ and $b(u, v)$ are the Dirichlet forms associated with $A$ and $B$ respectively. The following analogue of theorem 4.1 allows the application of the results of $\$ 3$ to this problem.

Theorem 4.15. Let $\Omega$ be a bounded open subset of $\mathbf{R}^{n}$ with locally Lipscbitzian boundary. Let $A$ be $x$ n operator of the form (4.1) satisfying (4.2), (4.10), (4.4) and $B$ an operator of the form (4.11) satisfying (4.12), (4.13). Let $Y$ be a $\sigma\left(\Pi L_{N_{a}}, \Pi E_{\bar{N}_{\alpha}}\right)$ closed subspace of $W^{m} L_{M}(\Omega)$ satisfying (4.14) and let $\left(Y, Y_{0} ; Z, Z_{0}\right)$ be the complementary system generated by $Y$ in $\left(\Pi L_{N a}, \Pi E_{N a}\right.$; $\Pi L_{\bar{N}_{a}}$, П $\left.E_{\bar{N}_{a}}\right)$. Let $T$ be the mapping from

$$
D(T)=\left\{u \in Y ; A_{\alpha}(\xi(u)) \in L_{\bar{N}}(\Omega) \text { for all }|\alpha| \leq m\right\}
$$

into $Z$ defined by $\langle v, T u\rangle=a(u, v)+b(u, v)$ for all $v \in Y_{0^{\circ}}$. Then $T$ is pseudomonotone with respect to any dense subspace $V$ of $Y_{0^{\circ}}$.

The following lemmas will be needed in the proof of Theorem 4.15 and later. Lemma 4.16 is a generalized version of the Vitali convergence theorem. Lemma 4.17 can be proved by standard arguments on Nemytskii operators as in [24, \$17] and by the method of Lemma 4.3. $\Omega$ is assumed to be bounded.

Lemma 4.16 (cf. [24, p. 99]). If the sequence $u_{n}(x) \in E_{N}(\Omega)$ converges a.e. in $\Omega$, then it converges in norm in $E_{N}(\Omega)$ if and only if the norms are uniformly absolutely continuous, i.e. for each $\epsilon>0$ there exists $\delta>0$ such that $\left\|u_{n} x_{\Omega}\right\|_{N}$ $<\epsilon$ for all $n$ and $\Omega^{\prime} \subset \Omega$ with meas $\left(\Omega^{\prime}\right)<\delta$.

Lemma 4.17. Let $g(x, u, v)=g\left(x, u_{1}, \cdots, u_{l}, v_{1}, \cdots, v_{\mathrm{J}}\right)$ be a real-valued function defined on $\Omega \times \mathbf{R}^{I} \times \mathbf{R}^{J}$ and satisfying the Carathéodory condition. Suppose that there exist $N$-functions $N$ and $N_{i}, a(x) \in L_{N}(\Omega), d(v) \in C\left(\mathbf{R}^{J}\right)$ and $c_{i} \in \mathbf{R}^{+}$sucb that, for all $x \in \Omega, u \in \mathbf{R}^{I}$ and $v \in \mathbf{R}^{J}$,

$$
|g(x, u, v)| \leq d(v)\left[a(x)+\sum_{i=1}^{I} N^{-1} N_{i}\left(c_{i} u_{i}\right)\right] .
$$

Then the mapping $(u(x), v(x)) \rightarrow g(x, u(x) v(x))$ sends $\Pi_{i} \mathcal{P}\left(E_{N_{i}}, 1 / c_{i}\right) \times \Pi_{j} L^{\infty}(\Omega)$ into $L_{N}(\Omega)$ and is finitely continuous to the $\sigma\left(L_{N}, E_{-}\right)$topology of $L_{N}(\Omega)$; it is uniformly bounded on each $\Pi_{i} B_{r_{i}}\left(0, L_{N_{i}}\right) \times \Pi_{j} B_{s_{j}}\left(0, L^{\infty}\right)$ when $r_{i}<1 / c_{i}$ for all $i=1, \ldots, I$; moreover it is continuous to the norm topology of $E_{p}(\Omega)$ when $P \ll N$. If we let $N(t)=t, N^{-1}(t)=t$ and $L_{N}(\Omega)=L^{1}(\Omega)$, then the above mapping sends 
$\Pi_{i} \mathcal{P}\left(E_{N_{i}}, 1 / c_{i}\right) \times \Pi_{j} L^{\infty}(\Omega)$ into $L^{1}(\Omega)$ and is continuous; it is uniformly bounded on each $\Pi_{i} B_{r_{i}}\left(0, L_{N_{i}}\right) \times \Pi_{j} B_{s_{j}}\left(0, L^{\infty}\right)$ when $r_{i}<1 / c_{i}$ for all $i=1, \ldots, l$.

Proof of Theorem 4.15. Clearly $T=T_{1}+T_{2}$ where $T_{1}$ is given by $D\left(T_{1}\right)=$ $D(T)$ and $\left\langle v, T_{1} u\right\rangle=a(u, v)$ for all $v \in Y_{0}$, and $T_{2}$ by $D\left(T_{2}\right)=Y$ and $\left\langle v_{0} T_{2} u\right\rangle=$ $b(u, v)$ for all $v \in Y_{0}$. By a simple generalization of Theorem 4.1, $T_{1}$ is pseudomonotone with respect to any dense subspace $V$ of $Y_{0}$. We will show that $T_{2}$ is completely continuous (cf. Remark 2.5), and then the conclusion of Theorem 4.15 will follow from Proposition 2.2.

Let $u_{i}$ be a bounded net in $Y$ such that $u_{i} \rightarrow u \in Y$ for $\sigma\left(Y, Z_{0}\right)$. By Propposition 4.13, $D^{\gamma} u_{i} \rightarrow D^{\gamma} u$ in $E_{P_{\gamma}}(\Omega)$ for $m-q(M) \leq|\gamma| \leq m-1$ and in $C(\bar{\Omega})$ for $|\gamma|<m-q(M)$, so that, by Lemmas 4.16 and $4.17, B_{\gamma}\left(\eta\left(u_{i}\right)\right) \rightarrow B_{\gamma}(\eta(u))$ in $E_{M_{y}}(\Omega)$ for $m-q(M) \leq|\gamma| \leq m-1$ and in $L^{1}(\Omega)$ for $|\gamma|<m-q(M)$, which implies $T_{2}\left(u_{i}\right) \rightarrow T_{2}(u)$ in $Z$. Q.E.D.

The existence theorems for (4.1) obtained in this section generalize results of Browder [3], [7]. Theorem 4.8 (with a lower order perturbation as in Theorem 4.15) also includes the result of Donaldson [12] where the conjugate $N$-functions are required to have to $\Delta_{2}$ property.

5. Applications II. We continue the study of systems of the form (4.1) with rapidly (or slowly) increasing coefficients and consider now the case where the $A_{a}$ 's satisfy the monotonicity conditions introduced by Leray-Lions [25].

Let $\Omega$ be a bounded open subset of $\mathbf{R}^{n}$ with locally Lipschitzian boundary. The basic conditions imposed on the coefficients $A_{a}$ are now, in addition to (4.2):

(5.1) There exist a $N$-function $M, N$-functions $N_{a}$ satisfying $M \prec N_{a}$ for $|a| \leq m, N_{a} \prec M$ for $|a|=m$ and $N_{a} \ll M_{a}$ for $m-q(M) \leq|a|<m, a_{a}(x) \epsilon$ $E_{\bar{N}_{a}}(\Omega)$ for $|a|=m, a_{a}(x) \in L_{\bar{N}_{a}}(\Omega)$ for $|a|<m$, a $N$-function $P$ satisfying $P \ll M, e \in C\left(\mathbf{R}^{s_{m-q}(M)-1}\right)$ and $c \in \mathbf{R}^{+}$such that for all $x \in \Omega$ and $\xi \in \mathbf{R}^{s_{m}}$ with component $\xi^{q}$ in $\mathbf{R}^{s_{m-q}(M)-1}$,

$$
\begin{aligned}
& \text { if }|a|=m, \\
& \left|A_{\alpha}(x, \xi)\right| \leq e\left(\xi^{q}\right)\left[a_{a}(x)+\sum_{|\beta|=m} \bar{N}_{a}^{-1} N_{\beta}\left(c \xi_{\beta}\right)+\sum_{m-q(M) \leq|\beta|<m} \bar{P}^{-1} N_{\beta}\left(c \xi_{\beta}\right)\right] ; \\
& \quad \text { if }|\alpha|<m, \\
& \left|A_{\alpha}(x, \xi)\right| \leq e\left(\xi^{q}\right)\left[a_{\alpha}(x)+\sum_{|\beta|=m} \bar{N}_{\alpha}^{-1} P\left(c \xi_{\beta}\right)+\sum_{m-q(M) \leq|\beta|<m} \bar{N}_{\alpha}^{-1} N_{\beta}\left(c \xi_{\beta}\right)\right] .
\end{aligned}
$$

(5.2) For each $x$ in $\Omega, \eta \in \mathbf{R}^{s_{m}-1}, \zeta$ and $\zeta^{\prime}$ in $\mathbf{R}^{s_{m}^{\prime}}$ with $\zeta \neq \zeta^{\prime}$,

$$
\sum_{|\alpha|=m}\left(A_{\alpha}(x, \zeta, \eta)-A_{\alpha}\left(x, \zeta^{\prime}, \eta\right)\right)\left(\zeta_{\alpha}-\zeta_{\alpha}^{\prime}\right)>0 \text {. }
$$


(5.3) For each $x$ in $\Omega, \zeta^{\prime}$ and $\zeta^{\prime \prime}$ in $\mathbf{R}^{s_{m}^{\prime}}$,

$$
\sum_{|a|=m}\left(A_{\alpha}\left(x, \zeta \eta \eta-\zeta_{\alpha}^{\prime}\right)\left(\zeta_{\alpha}-\zeta_{\alpha}^{\prime \prime}\right) \rightarrow+\infty\right.
$$

as $|\zeta| \rightarrow+\infty$ in $\mathbf{R}_{m}^{s_{m}^{\prime}}$, uniformly for bounded $\eta$ in $\mathbf{R}^{s_{m-1}}$

Let $Y$ be a $\sigma\left(\Pi L_{N a}, \Pi E_{\bar{N}}\right)$ closed subspace of $W^{m} L_{M}(\Omega)$ such that (4.14) holds. Let $f \in Y_{0}^{*}$. The V.B.V.P. for $A(u)=f$ witb respect to $Y$ asks for an element $u \in Y$ such that $A_{a}(\xi(u)) \in L_{\bar{N}_{a}}(\Omega)$ for all $|a| \leq m$ and $a(u, v)=f(v)$ for all $v \in Y_{0}$.

More generally we consider a one-parameter family of operators

$$
A_{t}(u) \equiv \sum_{|\alpha| \leq m}(-1)^{|a|} D^{\alpha} A_{\alpha}\left(x, u, \ldots, \nabla^{m} u, t\right)
$$

where $t \in[0,1]$. The coefficients $A_{\alpha}(x, \xi, t)$ are assumed to satisfy (4.2), (5.1), (5.2), (5.3) for each $t$; moreover it is assumed that they are continuous in $(\xi, t)$ for fixed $x$, that the functions $M, N_{a}, a_{a}(x), P, e$ and the constant $c$ of (5.1) can be chosen independently of $t$, and that the convergence in (5.3) is uniform in $t$. Briefly we will say that (4.2), (5.1), (5.2), (5.3) are satisfied uniformly in $t$.

The following analogue of Theorems 4.1 and 4.15 generalizes results of Leray-Lions [25] and Browder [7].

Theorem 5.1. Let $\Omega$ be a bounded open subset of $\mathbf{R}^{\boldsymbol{n}}$ with locally Lipschitzian boundary. Let $\left\{A_{t} ; t \in[0,1]\right\}$ be a one-parameter family of operators of the form (5.4) satisfying (4.2), (5.1), (5.2), (5.3) uniformly in t. Let $Y$ be a $\sigma\left(\Pi L_{N a}, \Pi E_{\bar{N}_{a}}\right.$ ) closed subspace of $W^{m} L_{M}(\Omega)$ satisfying (4.14) and let $\left(Y, Y_{0} ; Z, Z_{0}\right)$ be the complementary system generated by $Y$ in (II $L_{N a}, \Pi E_{N a}$; П $L_{\bar{N}_{a}}$, I $\left.E_{\bar{N}_{a}}\right)$. For each $t_{\text {, let }} T_{t}$ be the mapping from

$$
D\left(T_{t}\right)=\left\{u \in Y ; A_{\alpha}(\xi(u), t) \in L_{\bar{N}}(\Omega) \text { for all }|a| \leq m\right\}
$$

into $Z$ defined by $\left\langle u, T_{t} u\right\rangle=a_{t}(u, v)$ for all $v \in Y_{0}$, where $a_{t}(u, v)$ is the Diricblet form associated with $A_{t^{*}}$ Then $\left\{T_{t}: D\left(T_{t}\right) \subset Y \rightarrow Z ; t \in[0,1]\right\}$ is a sequentially pseudomonotone bomotopy with respect to any dense subspace $V$ of $Y_{0^{\circ}}$.

Remark 5.2. By means of an argument similar to that used at the beginning of the proof of Theorem 5.1 below, one can see that

$$
\begin{aligned}
D\left(T_{t}\right) & =\left\{u \in Y ; \sum_{|a| \leq m} A_{a}(\xi(u), t) D^{a} u \in L^{1}(\Omega)\right\} \\
& =\left\{u \in Y ; \sum_{|a|=m} A_{a}(\xi(u), t) D^{a} u \in L^{1}(\Omega)\right\} .
\end{aligned}
$$

Proof of Theorem 5.1. Let $V$ be a dense subspace of $Y_{n}$. By a simple 
generalization of Lemma 4.3, $V \subset D\left(T_{t}\right)$ for each $t$ and $T$ is finitely continuous from $[0,1] \times V$ to the $\sigma(Z, V)$ topology of $Z$. Let $\left(t_{i}, u_{i}\right)$ be a sequence with $u_{i} \in V, u_{i} \rightarrow u \in Y$ for $o\left(Y, Z_{0}\right), t_{i} \rightarrow t_{0} T_{t_{i}}\left(u_{i}\right) \rightarrow f \in Z$ for $\sigma(Z, V)$ and

$$
\lim \sup \left\langle u_{i}, T_{t_{i}}\left(u_{i}\right)\right\rangle \leq\langle u, f\rangle \text {. }
$$

We must prove that $u \in D\left(T_{t}\right), T_{t}(u)=f$ and $\left\langle u_{i}, T_{t_{i}}\left(u_{i}\right)\right\rangle \rightarrow\left\langle u_{i} f_{\%}\right.$. As usual, one can pass to a subsequence if necessary.

First we show that, for each $|a| \leq m, A_{a}\left(\xi\left(u_{i}\right), t_{i}\right)$ remains bounded in $L_{\bar{N}_{\alpha}}(\Omega)$. Since $C \prec \prec D$ implies that, for each $\epsilon>0, C(s) \leq D(\epsilon s)$ for $s \geq s_{\epsilon}$, it follows from (5.1) that when $|\alpha|<m$, for each $\epsilon>0$, there exists $K_{\epsilon}$ such that

$$
\left|A_{\alpha}(x, \xi, t)\right| \leq e\left(\xi^{q}\right)\left[a_{\alpha}(x)+K_{\epsilon}+\sum_{m-q(M) \leq|\beta| \leq m} \bar{N}_{a}^{-1} M_{\beta}\left(\epsilon \xi_{\beta}\right)\right]
$$

for all $x \in \Omega, \xi \in \mathbf{R}^{s_{m}}$ and $t \in[0,1]$. Thus, choosing $\epsilon$ sufficiently small, say $1 / \epsilon>\sup \left\{\left\|D^{\beta} u_{i}\right\|_{\left(M_{\beta}\right)} ; i=1,2, \ldots\right.$ and $\left.m-q(M) \leq|\beta| \leq m\right\}$, we deduce from Lemma 4.17 that $A_{a}\left(\xi\left(u_{i}\right), t_{i}\right),|\alpha|<m$, remains bounded in $L_{\bar{N}}(\Omega)$. To see that $A_{a}\left(\xi\left(u_{i}\right), t_{i}\right)$ also remains bounded in $L_{\bar{N}}(\Omega)$ when $|a|=m$, let $w=\left(w_{\beta}\right) \epsilon$ $\Pi_{|\beta|=m} E_{N \beta}(\Omega)$. By an argument similar to the preceding one, $A_{a}\left(\zeta(w), \eta\left(u_{i}\right), t_{i}\right)$, $|\alpha|=m$, remains bounded in $L_{\bar{N}_{\alpha}}(\Omega)$. We have, by (5.2),

$$
\left.\int_{\Omega|a|=m} \sum_{\alpha}\left(\xi\left(u_{i}\right), t_{i}\right)-A_{\alpha}\left(\zeta(w), \eta\left(u_{i}\right), t_{i}\right)\right)\left(D^{a} u_{i}-w_{\alpha}\right) d x \geq 0,
$$

i.e.

$$
\begin{aligned}
\int_{Q} & \sum_{|\alpha|=m} A_{\alpha}\left(\xi\left(u_{i}\right), t_{i}\right) w_{\alpha} d x \\
& \leq \int_{Q} \sum_{|\alpha| \leq m} A_{\alpha}\left(\xi\left(u_{i}\right), t_{i}\right) D^{\alpha} u_{i} d x \\
& -\int_{Q|a|<m} A_{a}\left(\xi\left(u_{i}\right), t_{i}\right) D^{\alpha} u_{i} d x+\int_{Q|a|=m} \sum_{\alpha}\left(\zeta(w), \eta\left(u_{i}\right), t_{i}\right)\left(w_{\alpha}-D^{a} u_{i}\right) d x .
\end{aligned}
$$

The first integral of the right-hand side remains bounded from above by (5.5), and the preceding discussion shows that the last two integrals remain bounded. Hence $A_{a}\left(\xi\left(u_{i}\right), t_{i}\right),|\alpha|=m$, remains bounded in $L_{\bar{N}_{a}}(\Omega)$ for $\sigma\left(L_{\bar{N}_{a}}, E_{N_{\alpha}}\right)$, i.e. remains bounded in $L_{\bar{N}}(\Omega)$.

Consequently, we can assume that, for each $|a| \leq m_{0} A_{a}\left(\xi\left(u_{i}\right), t_{i}\right) \rightarrow b_{a} \epsilon$ $L_{\bar{N}_{a}}(\Omega)$ for $\sigma\left(L_{\bar{N}_{a}} E_{N_{a}}\right)$. It follows that the linear form $f \in Z=Y_{0}^{*}$ can be identified to $\left(b_{a}\right) \in \Pi L_{\bar{N}_{a}}$. More precisely the action of $f$ over $V$ is clearly given by 


$$
\langle v, f\rangle=\int_{\Omega|a| \leq m} \sum_{\alpha} D^{a} v d x, \quad v \in V
$$

Since $V$ is dense in $Y_{0}$ and $Y_{0}$ satisfies (4.14), this formula also describes the action of $f$ over $Y$, i.e. holds for $v \in Y$. On the other hand, by Proposition 4.13, we can assume that $D^{a} u_{i} \rightarrow D^{a} u$ a.e. in $\Omega$ when $|a|<m$. We will show that this almost everywhere convergence also holds (for a subsequence) when $|\alpha|=m$. It will then follow from Lemma 4.4 that $A_{a}(\xi(u), t)=b_{a}$ for all $|a| \leq m$, i.e., that $u \in D\left(T_{t}\right)$ and $T_{t}(u)=f$.

Let $\Omega_{k}=\left\{x \in \Omega ;\left|D^{a} u(x)\right| \leq k\right.$ for all $\left.|\alpha|=m\right\}$ and denote by $\chi_{k}$ the characteristic function of $\Omega_{k}$. Clearly, $\Omega_{k} \subset \Omega_{k+1}$ and meas $\left(\Omega \backslash \Omega_{k}\right) \rightarrow 0$ as $k \rightarrow \infty$. Fix $k$ and let $l \geq k$. We have, by (5.2),

$$
\begin{aligned}
& \int_{\Omega_{k}} \sum_{|\alpha|=m}\left(A_{\alpha}\left(\zeta(u), \eta\left(u_{i}\right), t_{i}\right)-A_{\alpha}\left(\xi\left(u_{i}\right), t_{i}\right)\right)\left(D^{\alpha} u-D^{\alpha} u_{i}\right) d x \\
& \leq \int_{\Omega_{l}} \sum_{|\alpha|=m}\left(A_{\alpha}\left(\zeta(u), \eta\left(u_{i}\right), t_{i}\right)-A_{\alpha}\left(\xi\left(u_{i}\right), t_{i}\right)\right)\left(D^{\alpha} u-D^{\alpha} u_{i}\right) d x \\
& \leq \int_{\mathbf{Q}|\alpha|=m} \sum_{a}\left(A_{a}\left(\chi_{l} \zeta(u), \eta\left(u_{i}\right), t_{i}\right)-A_{\alpha}\left(\xi\left(u_{i}\right), t_{i}\right)\right)\left(\chi_{l} D^{\alpha} u-D^{a} u_{i}\right) d x \\
& =\int_{\sigma|\alpha|=\eta \eta} \sum_{\alpha}\left(\xi\left(u_{i}\right), t_{i}\right) D^{\alpha} u_{i} d x-\int_{Q|\alpha|=m} A_{\alpha}\left(\xi\left(u_{i}\right), t_{i}\right) \chi_{l} D^{\alpha} u d x \\
& +\int_{2|\alpha|=m} \sum_{\alpha}\left(\chi_{l} \zeta(u), \eta\left(u_{i}\right), t_{i}\right)\left(\chi_{l} D^{\alpha} u-D^{\alpha} u_{i}\right) d x \text {. }
\end{aligned}
$$

Going to the limit as $i \rightarrow+\infty$, we obtain for the first integral of the right-hand side

$$
\underset{i \rightarrow \infty}{\limsup } \int_{\Omega} \sum_{|\alpha|=m} A_{\alpha}\left(\xi\left(u_{i}\right), t_{i}\right) D^{a} u_{i} d x \leq \int_{\Omega|a|=m} \sum_{\alpha} D^{a} u d x
$$

because of (5.5) and

$$
\int_{\Omega} \sum_{|\alpha|<m} A_{\alpha}\left(\xi\left(u_{i}\right), t_{i}\right) D^{\alpha} u_{i} d x \rightarrow \int_{\Omega} \sum_{|\alpha|<m} b_{a} D^{\alpha} u d x
$$

(the last convergence holds since $A_{a}\left(\xi\left(u_{i}\right), t_{i}\right) \rightarrow b_{a}$ for $\sigma\left(L_{\bar{N}}, E_{N_{\alpha}}\right)$ and $D^{a} u_{i} \rightarrow D^{a} u$ in norm in $E_{N \alpha}(\Omega)$ when $\left.|a|<m\right)$. The second integral converges to $-\int_{\Omega} \Sigma_{|a|=m} b_{a} \chi_{l} D^{a} u d x$. Since, by Lemmas 4.16 and 4.17, $A_{a}\left(\chi_{l} \zeta(u), \eta\left(u_{i}\right), t_{i}\right)$ $\rightarrow A_{a}\left(\chi_{l} \zeta(u), \eta(u), t\right)$ in norm in $E_{\bar{N}_{a}}(\Omega)$ when $|a|=m$, the last integral also converges. Thus 


$$
\begin{gathered}
\limsup _{i \rightarrow \infty} \int_{\mathbf{Q}_{k}} \sum_{|\alpha|=m}\left(A_{\alpha}\left(\zeta(u), \eta\left(u_{i}\right), t_{i}\right)-A_{\alpha}\left(\xi\left(u_{i}\right), t_{i}\right)\right)\left(D^{\alpha} u-D^{\alpha} u_{i}\right) d x \\
\leq \int_{Q \backslash \Omega_{l}} \sum_{|\alpha|=m}\left(b_{\alpha}-A_{\alpha}(0, \eta(u), t)\right) D^{\alpha} u d x,
\end{gathered}
$$

and letting $l \rightarrow \infty$,

$$
\underset{i \rightarrow \infty}{\limsup } \int_{\Omega_{k}} \sum_{|a|=m}\left(A_{\alpha}\left(\zeta(u), \eta\left(u_{i}\right), t_{i}\right)-A_{\alpha}\left(\xi\left(u_{i}\right), t_{i}\right)\right)\left(D^{\alpha} u-D^{a} u_{i}\right) d x \leq 0
$$

Since the integrand is nonnegative by (5.2), it converges to 0 in $L^{1}\left(\Omega_{k}\right)$ as $i \rightarrow \infty$. On the other hand, by a previous argument,

$$
\sum_{|\alpha|=m}\left(A_{\alpha}(\xi(u), t)-A_{\alpha}\left(\zeta(u), \eta\left(u_{i}\right), t_{i}\right)\right)\left(D^{\alpha} u-D^{a} u_{i}\right) \rightarrow 0 \text { in } L^{1}\left(\Omega_{k}\right)
$$

as $i \rightarrow \infty$. Thus

$$
\sum_{|\alpha|=m}\left(A_{a}(\xi(u), t)-A_{\alpha}\left(\xi\left(u_{i}\right), t_{i}\right)\right)\left(D^{\alpha} u-D^{\alpha} u_{i}\right) \rightarrow 0 \text { in } L^{1}\left(\Omega_{k}\right) .
$$

This is true for each $k$, so that there is a subsequence such that

$$
\sum_{|a|=m}\left(A_{\alpha}(\xi(u), t)-A_{\alpha}\left(\xi\left(u_{i}\right), t_{i}\right)\right)\left(D^{a} u-D^{a} u_{i}\right) \rightarrow 0 \text { a.e. in } \Omega
$$

as $i \rightarrow \infty$. Now we can argue as in the classical case [25], [7], using the full force of conditions (5.2) and (5.3). It first follows from (5.8) and (5.3) that, for a.e. $x \in \Omega, \zeta\left(u_{i}\right)(x)$ remains bounded in $\mathbf{R}^{s_{m}}$. Taking, for a given $x^{0}$, a subsequence such that $\zeta\left(u_{i}\right)\left(x^{0}\right) \rightarrow \zeta^{0}$, we deduce from (5.8) that

$$
\sum_{|\alpha|=m}\left(A_{\alpha}\left(\xi(u)\left(x^{0}\right), t\right)-A_{\alpha}\left(\zeta^{0}, \eta(u)\left(x^{0}\right), t\right)\right)\left(D^{a} u\left(x^{0}\right)-\zeta_{\alpha}^{0}\right)=0,
$$

which implies, by (5.2), $\zeta^{0}=\zeta(u)\left(x^{0}\right)$. Therefore $\zeta\left(u_{i}\right)\left(x^{0}\right)$ converges for the original sequence to $\zeta(u)\left(x^{0}\right)$, and the almost everywhere convergence of $D^{\alpha} u_{i}$ to $D^{a} u,|a|=m$, is proved.

To complete the proof of Theorem 5.1, it remains to show that $\left\langle u_{i}, T_{t_{i}}\left(u_{i}\right)\right\rangle$ $\rightarrow\langle u, f\rangle$. Because of (5.7) and (5.6), it suffices to verify that

(5.9) $\liminf _{i \rightarrow \infty} \int_{\Omega} \sum_{|\alpha|=m} A_{\alpha}\left(\xi\left(u_{i}\right), t_{i}\right) D^{\alpha} u_{i} d x \geq \int_{\Omega|\alpha|=m} \sum_{\alpha}(\xi(u), t) D^{\alpha} u d x$.

Define $\Omega_{k}$ as above and fix $k$. We have, by (5.2),

$$
\left.\int_{\Omega|\alpha|=m} \sum_{a}\left(A_{k} \zeta(u), \eta\left(u_{i}\right), t_{i}\right)-A_{\alpha}\left(\xi\left(u_{i}\right), t_{i}\right)\right)\left(\chi_{k} D^{a} u-D^{a} u_{i}\right) d x \geq 0 .
$$

Going to the limit as above, 


$$
\begin{aligned}
& \liminf _{i \rightarrow \infty} \int_{\Omega} \sum_{|\alpha|=m} A_{\alpha}\left(\xi\left(u_{i}\right), t_{i}\right) D^{\alpha} u_{i} d x \\
& \geq-\int_{\Omega} \sum_{|a|=m}\left(A_{\alpha}\left(\chi_{k} \zeta(u), \eta(u), t\right)-A_{\alpha}(\xi(u), t)\right) \chi_{k} D^{a} u d x \\
& +\int_{\Omega} \sum_{|\alpha|=m} A_{\alpha}\left(\chi_{k} \zeta(u), \eta(u), t\right) D^{\alpha} u d x \\
& =\int_{\mathbf{Q}} \sum_{|\alpha|=m} A_{a}(\xi(u), t) D^{\alpha} u d x \\
& +\int_{\boldsymbol{Q} \backslash \boldsymbol{Q}_{k}} \sum_{|\alpha|=m}\left(A_{\alpha}(0, \eta(u), t)-A_{\alpha}(\xi(u), t)\right) D^{\alpha} u d x .
\end{aligned}
$$

Letting $k \rightarrow \infty$, we obtain (5.9). Q.E.D.

Remark 5.3. If the assumptions of Theorem 5.1 are verified and if $B$ is a perturbation of the form (4.11) satisfying (4.12) and (4.13), then $\left\{S_{t}: D\left(S_{t}\right) \subset Y\right.$ $\rightarrow Z ; t \in[0,1]\}$ defined by $D\left(S_{t}\right)=D\left(T_{t}\right)$ and $\left\langle v, S_{t} u\right\rangle=a_{t}(u, v)+b(u, v)$ for all $v \in Y_{0}$ is a sequentially pseudomonotone homotopy with respect to any dense subspace $V$ of $Y_{0}$. Note also that Theorem 5.1 applies in particular to a single operator $A$. In this case the arguments of the above proof show that the graph of $T$ is sequentially closed with respect to the convergence involved in Theorem 3.10 and 3.15. Finally note that these arguments also show that $\left\{T_{t}: D\left(T_{t}\right) \subset Y\right.$ $\rightarrow Z ; t \in[0,1]\}$ is strongly quasibounded on $Y$ with respect to any $y \in Y_{0^{\circ}}$

The combination of Theorem 5.1 with the results of $\$ 3$ leads to several existence theorems for (4.1). For instance:

Theorem 5.4. Let $\Omega$ be a bounded open subset of $\mathbf{R}^{n}$ with locally Lipschitzian boundary. Let $A$ be an operator of the form (4.1) satisfying (4.2), (5.1), (5.2) and (5.3). Let $Y$ be a $\sigma\left(\Pi L_{N a}\right.$, $\left.\Pi E_{\bar{N}_{\alpha}}\right)$ closed subspace of $W^{m} L_{M}(\Omega)$ satisfying (4.14). Suppose that $a(u, u)\|u\|_{m_{0}(M)}^{-1} \rightarrow+\infty$ as $\|u\|_{m_{0}(M)} \rightarrow+\infty$ in $Y_{0}$. Then for each $\sigma\left(\Pi E_{N_{a}}\right.$, II $E_{\bar{N}_{a}}$ ) continuous linear form $f$ on $Y_{0}$, the V.B.V.P. for $A(u)=f$ with respect to $Y$ bas at least one solution.

Theorem 5.5. Let $\Omega$ be a bounded open subset of $\mathbf{R}^{\boldsymbol{n}}$ with locally Lipscbitzian boundary. Let $\left\{A_{t} ; t \in[0,1]\right\}$ be a one-parameter family of operators of the form (5.4) satisfying (4.2), (5.1), (5.2), (5.3) uniformly in $t$. Let $Y$ be a $\sigma\left(\Pi L_{N \alpha}\right.$ П $E_{\bar{N}_{a}}$ ) closed subspace of $W^{m} L_{M}(\Omega)$ satisfying (4.14). Suppose that $A_{1}$ is odd and that for each $\sigma\left(\Pi E_{N a}, \Pi E_{\bar{N}_{a}}\right)$ continuous linear form $f$ on $Y_{0}$ there exist a constant $K$ and a neigbbourbood $\pi$ of $f$ in $Y_{0}^{*}$ such that, for any $g \in \boldsymbol{H}$, any $t \in[0,1]$ and any solution $u$ of the V.B.V.P. for $A_{t}(u)=g$ with respect to $Y,\|u\|_{m_{0}(M)} \leq K$. Then for each $t \in[0,1]$ and each $\sigma\left(\Pi E_{N_{\alpha}} \Pi E_{\bar{N}_{a}}\right)$ 
continuous linear form $f$ on $Y_{0}$, the V.B.V.P. for $A_{t}(u)=f$ with respect to $Y$ bas at least one solution.

Concrete analytical conditions on the coefficients $A_{a}$ implying coercivity or a local a priori bound can be deduced from Lemma 3.14 or from the treatment of the following example respectively.

Example 5.6. Consider the Dirichlet problem for the operator $P(u)+A(u)$,

$$
\begin{aligned}
P(u) \equiv & \sum_{|a|=m}(-1)^{|a|} D^{a}\left(p\left(D^{a} u\right)\right), \\
A(u) \equiv & \sum_{|a|=m}(-1)^{|a|} D^{a} A_{\alpha}\left(x, u, \cdots, \nabla^{m-1} u\right) \\
& +\left.\sum_{|a|<m}(-1)^{|a|}\right|^{a} A_{\alpha}\left(x, u, \cdots, \nabla^{m} u\right),
\end{aligned}
$$

on a bounded open subset $\Omega$ of $\mathbf{R}^{n}$ with locally Lipschitzian boundary. Here $p: \mathbf{R} \rightarrow \mathbf{R}$ is a strictly increasing odd continuous function with $p(+\infty)=+\infty$. Write $M(t)=\int_{0}^{t} p(s) d s$. It is assumed that the $A_{a}^{\prime}$ 's satisfy the growth condition (5.1) with $N_{a}=M$ for simplicity and the sign condition

$$
\sum_{|a| \leq m} A_{a}(x, \xi) \xi_{a} \geq 0
$$

for all $\xi \in \mathbf{R}^{s_{m}}$. To apply Theorem 5.5, we consider the one-parameter family $A_{t}(u)=P(u)+(1-t) A(u), t \in[0,1]$. It is easily verified that (4.2), (5.1), (5.2), (5.3) hold uniformly in $t$. Clearly, $A_{1}$ is odd. As before, $Y=W_{0}^{m} L_{M}(\Omega)$ satisfies (4.5), $Y_{0}=W_{0}^{m} E_{M}(\Omega), Y_{0}^{*}=Z=W^{-m} L_{\bar{M}}(\Omega)$ and the subspace $Z_{0}$ of $Y_{0}^{*}$ consisting of those linear forms on $Y_{0}$ which are $\sigma\left(\Pi E_{M}, \Pi E_{\bar{M}}\right)$ continuous in $W^{-m} E_{\bar{M}}(\Omega)$. To verify the local a priori bound, let $f=\left(f_{\alpha}\right) \in Z_{0^{*}}$. Let $r>1$, choose $c$ such that $\int_{\Omega} \bar{M}\left(r f_{\alpha}\right) d x \leq c$ for all $|\alpha| \leq m$, and define

$$
r=\left\{g=\left(g_{a}\right) \in Z ; \int_{Q} \bar{M}\left(r g_{a}\right) \leq c+1 \text { for all }|a| \leq m\right\} \text {. }
$$

$r$ is a norm neighbourhood of $f$ in $Z$ (cf. Example 4.11). We claim that if $r$ is taken large enough, then the possible solutions of the V.B.V.P. for $A_{t}(u)=g$ with respect to $Y$ remain bounded in $Y$ as $g \in \boldsymbol{r}$ and $t \in[0,1]$. Indeed, if $u$ is such a solution, then we obtain as in Example 4.11, using (5.10),

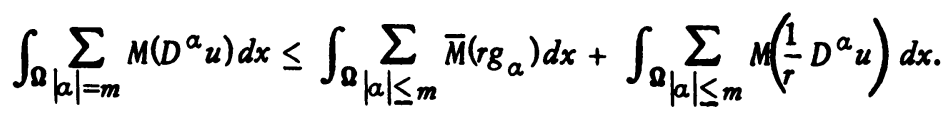

Now, by Poincare's inequality (Lemma 5.7 below), the last integral of the righthand side is less than 


$$
\frac{1}{r} \int_{\boldsymbol{\Omega}} \sum_{|a|=m} M\left(D^{a} u\right) d x+c_{m} \int_{\Omega_{|a|=m}} \sum_{\left(\frac{c_{m, \boldsymbol{Q}}}{r} D^{a} u\right) d x}
$$

Consequently a bound on $\int_{\Omega} \Sigma_{|a|=m} M\left(D^{a} u\right) d x$ can be derived provided $r>c_{m, \Omega}$ and $r>1+c_{m} c_{m, \mathbb{R}}$. It follows that $D^{a} u,|\alpha|=m$, remains bounded in $L_{M}(\Omega)$. Hence, by Poincaré's inequality (Corollary 5.8 below) $u$ remains bounded in $W_{0}^{m} L_{M}(\Omega)$. Theorem 5.5 can thus be applied: for each $f \in W^{-m} E_{\bar{M}}(\Omega)$, the V.B.V.P. for $P(u)+A(u)=f$ with respect to $W_{0}^{m} L_{M}(\Omega)$ has at least one solution.

Lemma 5.7. Let $\Omega$ be a bounded open subset of $\mathbf{R}^{n}$. Then there exist constants $c_{m}$ and $c_{m, Q}$ such that

$$
\int_{\Omega|a|<m} M\left(D^{a} u\right) d x \leq c_{m} \int_{\Omega} \sum_{|a|=m} M\left(c_{m, \Omega} D^{a} u\right) d x
$$

for all $u \in W_{0}^{m} L_{M}(\Omega)$

Proof. It suffices to show that

$$
\int_{Q} M(u) d x \leq \int_{\Omega} M\left(2 d \frac{\partial u}{\partial x_{1}}\right) d x
$$

for all $u \in W_{0}^{1} L_{M}(\Omega)$, where $d$ is the diameter of $\Omega$. First suppose that $u \in \mathscr{D}(\Omega)$. Then

$$
\begin{aligned}
M\left(u\left(x_{1}, x_{2}, \cdots, x_{n}\right)\right) & =M\left(\int_{-\infty}^{x_{1}} \frac{\partial u}{\partial x_{1}}\left(\xi, x_{2}, \ldots, x_{n}\right) d \xi\right) \\
& \leq \frac{1}{d} \int_{-\infty}^{+\infty} M\left(d \frac{\partial u}{\partial x_{1}}\left(\xi, x_{2}, \cdots, x_{n}\right)\right) d \xi,
\end{aligned}
$$

and thus

$$
\int_{\Omega} M(u(x)) d x \leq \int_{\Omega} M\left(d \frac{\partial u}{\partial x_{1}}(x)\right) d x .
$$

Now suppose that $u \in W_{0}^{1} L_{M}(\Omega)$ has compact support in $\Omega$ and consider its regularized function $u_{\epsilon}$ (cf. Lemma 1.6). Then (5.12) holds for $u_{\epsilon}$ :

$$
\int_{\mathbf{Q}} M\left(u_{\epsilon}(x)\right) d x \leq \int_{\mathbf{Q}^{M}}\left(d\left(\frac{\partial u}{\partial x_{1}}\right)_{\epsilon}(x)\right) d x .
$$

Since $u_{\epsilon} \rightarrow u$ for $\sigma\left(L_{M}, E_{\bar{M}}\right)$ as $\epsilon \rightarrow 0$ and since the functional $a(x) \mapsto$ $\int_{\Omega} M(a(x)) d x$ is $\sigma\left(L_{M}, E_{\bar{M}}\right)$ lower semicontinuous on $L_{M}(\Omega)$ (because it is the convex functional conjugate to $b(x) \mapsto \int_{\Omega} \bar{M}(b(x)) d x$ on $E_{\bar{M}}(\Omega)$, cf. [29, p. 220]),

$$
\int_{\Omega} M(u(x)) d x \leq \liminf _{\epsilon \rightarrow 0} \int_{\Omega} M\left(u_{\epsilon}(x)\right) d x .
$$

On the other hand, writing $d \partial u / \partial x_{1}=v$, we have 


$$
\begin{aligned}
\int_{\Omega} M\left(v_{\epsilon}(x)\right) d x & =\int_{\Omega} M\left[\int_{\mathbf{R}^{n}} v(x-y) \phi_{\epsilon}(y) d y\right] d x \\
& \leq \int_{\Omega} \int_{\mathbf{R}^{n}} M(v(x-y)) \phi_{\epsilon}(y) d y d x=\int_{\Omega}(M(v))_{\epsilon}(x) d x .
\end{aligned}
$$

If $\int_{\Omega} M(v) d x=+\infty$, then (5.12) holds trivially for $u$. If $\int_{\Omega} M(v) d x<+\infty$, then $(M(v))_{\epsilon} \rightarrow M(v)$ in $L^{1}(\Omega)$ as $\epsilon \rightarrow 0$, and consequently (5.12) holds for $u$. Finally, let $u$ be arbitrary in $W_{0}^{1} L_{M}(\Omega)$ and consider an open set $\Omega^{\prime} \supset \bar{\Omega}$ with diameter less than $2 d$. Then the function $u$ obtained by extending $u$ by zero outside $\Omega$ belongs to $W_{0}^{1} L_{M}\left(\Omega^{\prime}\right)$ and has compact support in $\Omega^{\prime}$, so that

$$
\int_{\mathbf{Q}^{\prime}}, M(u) d x \leq \int_{\mathbf{Q}^{\prime}} M\left(2 d \frac{\partial u}{\partial x_{1}}\right) d x
$$

which implies (5.11). Q.E.D.

Corollary 5.8. Let $\Omega$ be a bounded open subset of $\mathbf{R}^{n}$. Then the two norms

$$
\left(\sum_{|a| \leq m}\left\|D^{\alpha} u\right\|_{(M)}^{2}\right)^{1 / 2} \text { and }\left(\sum_{|\alpha|=m}\left\|D^{\alpha} u\right\|_{(M)}^{2}\right)^{1 / 2}
$$

are equivalent on $W_{0}^{m} L_{M}(\Omega)$.

Proof. If $D^{a} u$ remains bounded in $L_{M}(\Omega)$ for each $|a|=m$, then for some $k>0, \int_{\Omega} M\left(D^{a} u / k\right) d x \leq 1$ for each $|a|=m$. By Lemma 5.7,

$$
\int_{\mathbf{a}} \sum_{|a|<m} M\left(D^{a} u / k c_{m, \mathbf{\Omega}}\right) d x \leq c_{m} c^{\prime},
$$

which implies that $D^{a} u$ remains bounded in $L_{M}(\Omega)$ for each $|a|<m$. Q.E.D.

The existence theorems for (4.1) obtained in this section generalize results of Leray-Lions [25] and Browder [7], [8]. Theorem 5.4 (and its anisotropic variant, cf. introduction to $\$ 4$ and Example 4.12) also includes the result announced recently by Fougères [15] where the conjugate $N$-functions are required to have the $\Delta_{2}$ property.

\section{BIBLIOGRAPHY}

1. S. Agmon, Lectures on elliptic boundary value problems, Van Nostrand Math. Studies, no. 2, Van Nostrand, Princeton, N. J., 1965. MR 31 \#2504.

2. H. Brézis, Equations et inéquations non linéaires dans les espaces vectoriels en dualité, Ann. Inst. Fourier (Grenoble) 18 (1968), fasc. 1, 115-175. MR 42 \#113.

3. F. E. Browder, Nonlinear elliptic boundary value problems, Bull. Amer. Math. Soc. 69 (1963), 862-874. MR 27 \#6048.

4. Nonlinear elliptic functional equations in a nonreflexive Banach space, Bull. Amer. Math. Soc. 72 (1966), 89-95. MR 32 \#2755.

5. - Nonlinear maximal monotone operators in Banach spaces, Math. Ann. 175 (1968), 89-113. MR 36 \#6989.

6. Nonlinear operators and nonlinear equations of evolution in Banach spaces, Proc. Sympos. Pure Math., vol. 18, part 2, Amer. Math. Soc., Providence, R.I. (to appear). 
7. F. E. Browder, Existence theorems for nonlinear partial differential equations, Proc. Sympos. Pure Math., vol. 16, Amer. Math. Soc., Providence, R.I., 1970, pp. 1-60. MR 42 \# 4855.

8. Nonlinear elliptic boundary value problems and the generalized topological degree, Bull. Amer. Math. Soc. 76 (1970), 999-1005. MR 41 \#8818.

9. - Existence theory for boundary value problems for quasilinear elliptic systems with strongly nonlinear lower order terms, Proc. Sympos, Pure Math,, vol. 23, Amer. Math Soc., Providence, R.I., 1973, pp. 269-286.

10. - Nonlinear elliptic eigenvalue problems with strong lower order nonlinearities, Rocky Mountain. Math. J. (to appear).

11. F. E. Browder and P. Hess, Nonlinear mappings of monotone type in Banach spaces, J. Functional Analysis 11 (1972), 251-294.

12. T. Donaldson, Nonlinear elliptic boundary value problems in Orlicz-Sobolev spaces, J. Differential Equations 10 (1971), 507-528.

13. T. Donaldson and N. S. Trudinger, Orlicz-Sobolev spaces and imbedding theorems, J. Functional Analysis 8 (1971), 52-75.

14. N. Dunford and J. T. Schwartz, Linear operators. I: General theory, Pure and Appl. Math., vol. 7, Interscience, New York, 1958. MR 22 \#8302.

15. A. Fougères, Opérateurs elliptiques du calcul des variations à coefficients très fortement non linéaires, C. R. Acad. Sci. Paris Sér. A-B 274 (1972), A763-A766.

16. J.-P. Gossez, Opérateurs monotones non linéaires dans les espaces de Banach non réflexifs, J. Math. Anal. Appl. 34 (1971), 371-395.

17. - On the range of a coercive maximal monotone operator in a nonreflexive Banach space, Proc. Amer. Math. Soc. 35 (1972), 88-92.

18. - On the subdifferential of a saddle function, J. Functional Analysis 11 (1972), 220-230.

19. - Boundary value problems for quasilinear elliptic equations with rapidly increasing coefficients, Bull. Amer. Math. Soc. 78 (1972), 753-758.

20. A. Granas, Sur la notion de degré topologique pour une certains classe de transformations multivalentes dans les espaces de Banach, Bull. Acad. Polon. Sci. Sér. Sci. Math. Astronom. Phys. 7 (1959), 191-194. MR 21 \#7457.

21. - Theorem on antipodes and theorems on fixed points for a certain class of multivalued mappings in Banach spaces, Bull. Acad. Polon. Sci. Sér. Sci. Math. Astronom. Phys. 7 (1959), 271-275. MR 22 \#8365.

22. P. Hess, On nonlinear mappings of monotone type with respect to two Banach spaces, J. Math. Pures Appl. 52 (1973), 13-26.

23. - A strongly nonlinear elliptic boundary value problem, J. Math. Anal. Appl. (to appear).

24. M. Krasnosel'skiǐ and Ju. V. Rutickǐ,, Convex functions and Orlicz spaces, GITTL, Moscow, 1958; English transl., Internat. Monographs on Advanced Math. Phys., Hindustan, Delhi; Noordhoff, Groningen, 1961. MR 23 \# A4016; MR 21 \#5144.

25. J. Leray and J.-L. Lions, Quelques résultats de Višik sur les problèmes elliptiques non linéaires par les méthodes de Minty-Browder, Bull. Soc. Math. France 93 (1965), 97-107. MR 33 \# 2939.

26. J. Lindenstrauss, On nonseparable reflexive Banach spaces, Bull. Amer. Math. Soc. 72 (1966), 967-970. MR 34 \# 4875.

27. J.-L. Lions, Quelques méthodes de résolution des problèmes aux limites non linéaires, Gauthier-Villars, Paris, 1969. MR 41 \# 4326.

28. W. Luxemburg, Banach function spaces, Thesis, Technische Hogeschool te Delft, 1955. MR 17, 285.

29. W. Luxemburg and A. Zaanen, Conjugate spaces of Orlicz spaces, Nederl.

Akad. Wetensch. Proc. Ser. A 59 = Indag. Math. 18 (1956), 217-228. MR 17, 1113.

30. G. J. Minty, Monotone (nonlinear) operators in a Hilbert space, Duke Math. J. 29 (1962), 341-346. MR 29 \#6319.

31. R. T. Rockafellar, On the maximal monotonicity of subdifferential mappings, Pacific J. Math. 33 (1970), 209-216. MR 41 \# 7432. 
32. R. T. Rockafellar, Local boundedness of nonlinear monotone operators, Michigan Math J. 16 (1969), 397-407. MR 40 \#6229.

33. - On the maximality of sums of nonlinear monotone operators, Trans. Amer. Math. Soc. 149 (1970), 75-88. MR 43 \#7984.

34. M. I. Višik, Quasilinear strongly elliptic systems of differential equations in divergence form, Trudy Moskov. Mat. Obšč. 12 (1963), 125-184 = Trans. Moscow Math. Soc. 1963, 140-208. MR 27 \#6017.

35. Solvability of the first boundary value problem for quasilinear equations with rapidly increasing coefficients in Orlicz classes, Dokl. Akad. Nauk SSSR 151 (1963), 758-761 = Soviet Math. Dokl. 4 (1963), 1060-1064. MR 27 \# 5032.

DÉPARTEMENT DE MATHÉMATIQUE, UNIVERSITÉ LIBRE DE BRUXELLES, BRUXELLES, BELGIUM 